\title{
Spectra of eigenstates in fermionic tensor quantum mechanics
}

\author{
Igor R. Klebanov, ${ }^{1,2}$ Alexey Milekhin, ${ }^{1}$ Fedor Popov, ${ }^{1}$ and Grigory Tarnopolsky ${ }^{3}$ \\ ${ }^{1}$ Department of Physics, Princeton University, Princeton, New Jersey 08544, USA \\ ${ }^{2}$ Princeton Center for Theoretical Science, Princeton University, Princeton, New Jersey 08544, USA \\ ${ }^{3}$ Department of Physics, Harvard University, Cambridge, Massachusetts 02138, USA
}

(Received 2 April 2018; published 31 May 2018)

\begin{abstract}
We study the $O\left(N_{1}\right) \times O\left(N_{2}\right) \times O\left(N_{3}\right)$ symmetric quantum mechanics of 3-index Majorana fermions. When the ranks $N_{i}$ are all equal, this model has a large $N$ limit which is dominated by the melonic Feynman diagrams. We derive an integral formula which computes the number of group invariant states for any set of $N_{i}$. It is non-vanishing only when each $N_{i}$ is even. For equal ranks the number of singlets exhibits rapid growth with $N$ : it jumps from 36 in the $O(4)^{3}$ model to 595354780 in the $O(6)^{3}$ model. We derive bounds on the values of energy, which show that they scale at most as $N^{3}$ in the large $N$ limit, in agreement with expectations. We also show that the splitting between the lowest singlet and non-singlet states is of order $1 / N$. For $N_{3}=1$ the tensor model reduces to $O\left(N_{1}\right) \times O\left(N_{2}\right)$ fermionic matrix quantum mechanics, and we find a simple expression for the Hamiltonian in terms of the quadratic Casimir operators of the symmetry group. A similar expression is derived for the complex matrix model with $S U\left(N_{1}\right) \times S U\left(N_{2}\right) \times$ $U(1)$ symmetry. Finally, we study the $N_{3}=2$ case of the tensor model, which gives a more intricate complex matrix model whose symmetry is only $O\left(N_{1}\right) \times O\left(N_{2}\right) \times U(1)$. All energies are again integers in appropriate units, and we derive a concise formula for the spectrum. The fermionic matrix models we studied possess standard 't Hooft large $N$ limits where the ground state energies are of order $N^{2}$, while the energy gaps are of order 1.
\end{abstract}

DOI: 10.1103/PhysRevD.97.106023

\section{INTRODUCTION AND SUMMARY}

In recent literature there has been considerable interest in the quantum mechanical models where the degrees of freedom are fermionic tensors of rank 3 or higher [1,2]. These models have solvable large $N$ limits dominated by the so-called melonic diagrams. Such novel large $N$ limits were discovered and developed in [3-11], mostly in the context of zero-dimensional tensor models with multiple $U(N)$ or $O(N)$ symmetries (for reviews, see [12-14]). The quantum mechanical tensor models are richer: they have interesting spectra of energy eigenstates and may have connections with physical systems like the quantum dots. More amibitiously, large $N$ tensor quantum mechanics may provide a dual description of two-dimensional black holes [15-18], in the sense of the gauge/gravity duality [19-21]. The original motivation [1] for introducing the tensor quantum mechanics is that they have a large $N$ limit similar to the one in the Sachdev-Ye-Kitaev (SYK) model [22-25], but without the necessity of the disorder. Indeed,

Published by the American Physical Society under the terms of the Creative Commons Attribution 4.0 International license. Further distribution of this work must maintain attribution to the author(s) and the published article's title, journal citation, and DOI. Funded by SCOAP ${ }^{3}$. as shown explicitly in [2], the 2- and 4-point functions in the large $N$ tensor models are governed by the same Schwinger-Dyson equations as were derived earlier for the SYK-like models [25-29].

At the same time, there are significant differences between the tensor and SYK-like models. An early hint was the different scaling of the corrections to the large $N$ limit [1] (see also the further work in [30-33]); more recently, additional evidence for the differences is emerging in the operator spectra and Hagedorn transition [34-36]. The formal structure of the two types of models is indeed quite different: the SYK-like models containing a large number of fermions, $N_{\mathrm{SYK}}$, have no continuous symmetries (although an $O\left(N_{\mathrm{SYK}}\right)$ symmetry appears in the replica formalism), while in the tensor models one typically encounters multiple symmetry groups. For example, in the Gurau-Witten (GW) model [1] containing 4 Majorana rank-3 tensors, the symmetry is $O(N)^{6}$; there is evidence $[30,34]$ that this model is the tensor counterpart of a 4-flavor generalization of the SYK model introduced in [29]. A simpler tensor quantum mechanics with a single rank3 Majorana tensor has $O(N)^{3}$ symmetry [2] and is the tensor counterpart of the basic SYK model with real fermions. The quantum mechanics of complex rank-3 fermionic tensor, which has $S U(N)^{2} \times O(N) \times U(1)$ symmetry [2], is the tensor counterpart of the variant of SYK model where real fermions are replaced by complex ones [37]. 
The absence of disorder and the presence of the continuous symmetry groups in the tensor models endows them with a number of theoretical advantages, but also makes them quite difficult to study. In the tensor models any invariant operator should be meaningful and be assigned a definite scaling dimension in the large $N$ limit. While the simplest scaling dimensions coincide with those in the corresponding SYK-like models, the operator spectrum in tensor models is much richer: the number of $2 k$-particle operators grows as $2^{k} k$ ! [34-36].

Beyond the operator spectrum, it is interesting to investigate the spectrum of eigenstates of the Hamiltonian. While this spectrum is discrete and bounded for finite $N$, the low-lying states become dense for large $N$ leading to the (nearly) conformal behavior where it makes sense to calculate the operator scaling dimensions. In the SYK model, the number of states is $2^{N_{\mathrm{SYK}} / 2}$, and numerical calculations of spectra have been carried out for rather large values of $N_{\text {SYK }}[38,39]$. They reveal a smooth distribution of energy eigenvalues, which is almost symmetric under $E \rightarrow-E$; it exhibits little sensitivity to the randomly chosen coupling constants $J_{i j k l}$. Such numerical studies of the SYK model have revealed various interesting physical phenomena, including the quantum chaos.

The corresponding studies of spectra in the GW model [1] and the $O(N)^{3}$ model [2] have been carried out in [40-46], but in these cases the numerical limitations have been more severe - the number of states grows as $2^{N^{3} / 2}$ in the $O(N)^{3}$ model and as $2^{2 N^{3}}$ in the GW model. This is why only the $N=2 \mathrm{GW}$ model and $N=2,3 O(N)^{3}$ models have been studied explicitly so far. ${ }^{1}$ Furthermore, in the tensor models the states need to be decomposed into various representations of the symmetry groups. As a result, the details of the energy spectrum in the $O(N)^{3}$ tensor model are quite different from those in the corresponding SYK model with $N_{\mathrm{SYK}}=N^{3}$ fermion species.

The goal of this paper is to improve our understanding of energy spectra in the tensor models. We will mostly focus on the simplest tensor model with $O(N)^{3}$ symmetry [2] and its generalization to $O\left(N_{1}\right) \times O\left(N_{2}\right) \times O\left(N_{3}\right)$, where the Majorana tensor degrees of freedom are $\psi^{a b c}$ with $a=1, \ldots, N_{1} ; b=1, \ldots, N_{2} ; c=1, \ldots, N_{3}$, and anticommutation relations

$$
\left\{\psi^{a b c}, \psi^{a^{\prime} b^{\prime} c^{\prime}}\right\}=\delta^{a a^{\prime}} \delta^{b b^{\prime}} \delta^{c c^{\prime}}
$$

The Hamiltonian is taken to be of the "tetrahedral" form $[2,10]$

\footnotetext{
${ }^{1}$ In [46] the exact values of the 140 singlet energies in the $O(2)^{6} \mathrm{GW}$ model were found to square to integers. Due to the discrete symmetries of the GW model, there are only 5 distinct $E<0$ eigenvalues (the singlet spectrum also contains 50 zeroenergy states). For these reasons the singlet spectrum of the $O(2)^{6} \mathrm{GW}$ model exhibits significant gaps.
}

$H=\frac{g}{4} \psi^{a b c} \psi^{a b^{\prime} c^{\prime}} \psi^{a^{\prime} b c^{\prime}} \psi^{a^{\prime} b^{\prime} c}-\frac{g}{16} N_{1} N_{2} N_{3}\left(N_{1}-N_{2}+N_{3}\right)$,

and we have added a shift to make the spectrum traceless. In Sec. II we discuss some essential features of this model, including its discrete symmetries. In Sec. III we will derive lower bounds on the energy in each representation of $S O\left(N_{1}\right) \times S O\left(N_{2}\right) \times S O\left(N_{3}\right)$. We will show that, in the melonic large $N$ limit where $g N^{3 / 2}=J$ is kept constant, the most stringent bounds (3.25) scale as $J N^{3}$, in agreement with expectations for a system with $N^{3}$ degrees of freedom. On the other hand, the splitting between lowest states in different representations is found to be of order $J / N$. Another derivation of this fact, based on effective action considerations, is presented in Sec. IV. While this gap vanishes in the large $N$ limit, we expect the splitting between states in the same representation to vanish much faster, i.e. as $c^{-N^{3}}$, where $c$ is a positive constant. Such small singlet sector gaps are needed to account for the large low-temperature entropy, which is given by the sum over melonic graphs and, therefore, has to be of order $N^{3}$.

If the global symmetry of the quantum mechanical model is gauged, this simply truncates the spectrum to the $S O\left(N_{1}\right) \times S O\left(N_{2}\right) \times S O\left(N_{3}\right)$ invariant states. In Sec. V we derive integral formulae for the number of singlets as functions of the ranks $N_{i}$. They lead to the conclusion that the singlets are present only when all $N_{i}$ are even. The absence of singlets when some of $N_{i}$ are odd can often be related to anomalies, which we discuss in Sec. V B. For the $O(N)^{3}$ model, the number of singlet states is shown in Table 1; it exhibits rapid growth from 2 for $N=2$, to 36 for $N=4$, to 595354780 for $N=6$. Thus, even though the $O(4)^{3}$ model is out of reach of complete numerical diagonalization because it has 64 Majorana fermions, in contrast to the SYK model with $N_{\mathrm{SYK}}=64$, it is far from the nearly conformal large $N$ limit. Indeed, since the spectrum is symmetric under $E \rightarrow-E$ [34], the number of distinct singlet eigenvalues with $E<0$ cannot exceed 18. Therefore, there are significant gaps in the singlet spectrum of the $O(4)^{3}$ model. On the other hand, the presence of the vast number of singlet states for the $O(6)^{3}$ model suggests that the low-lying singlet spectrum should be dense for $N=6$ and higher. For large $N$ the number of singlets grows as $\exp \left(N^{3} \log 2 / 2-3 N^{2} \log N / 2\right)$. Since all of these states must fit in an energy interval of order $N^{3}$,

TABLE I. Number of singlet states in the $O(N)^{3}$ model.

\begin{tabular}{cc}
\hline \hline$N$ & No. of singlet states \\
\hline 2 & 2 \\
4 & 36 \\
6 & 595354780 \\
\hline \hline
\end{tabular}


it is plausible that the gaps between low-lying singlet states vanish as $c^{-N^{3}}$.

The $O\left(N_{1}\right) \times O\left(N_{2}\right) \times O\left(N_{3}\right)$ tensor model (1.2) may be viewed as $N_{3}$ coupled Majorana $N_{1} \times N_{2}$ matrices [47,48]. As discussed in Sec. VI A, for $N_{3}=1$ we find a one-matrix model with $O\left(N_{1}\right) \times O\left(N_{2}\right)$ symmetry, which is exactly solvable because the Hamiltonian may be written in terms of a quadratic Casimir. When we set $N_{3}=2$ we find a complex $N_{1} \times N_{2}$ matrix model with $O\left(N_{1}\right) \times O\left(N_{2}\right) \times U(1)$ symmetry. It may be studied numerically for values of $N_{1}$ and $N_{2}$ as large as 4 and reveals a spectrum which is integer in units of $g / 4$. In Sec. VIC we explain why this fermionic matrix model is again exactly solvable and derive a concise expression (6.23) for its spectrum. When both $N_{1}$ and $N_{2}$ are even, so that the spectrum contains singlet states, we show that the ground state is a singlet. In Sec. VI B we apply similar methods to another complex matrix model, which was introduced in [49] and has $S U\left(N_{1}\right) \times S U\left(N_{2}\right) \times U(1)$. It is the $N_{3}=1$ case of the complex tensor quantum mechanics with $S U\left(N_{1}\right) \times S U\left(N_{2}\right) \times O\left(N_{3}\right) \times U(1)$ symmetry [2]. We show that the Hamiltonian of this model may be expressed in terms of the symmetry charges. The solvable matrix models presented in Sec. VI have standard 't Hooft limits when $N_{1}=N_{2}=N$ is sent to infinity while $\lambda=g N$ is held fixed. Then the low-lying states have energies $\sim \lambda N^{2}$, while the splittings are of order $\lambda$. So, in contrast to the melonic large $N$ limit, the energy levels do not become dense in the " $t$ Hooft limit of the matrix models. Nevertheless, these fermionic matrix models are nice examples of exactly solvable 't Hooft limits.

\section{THE RANK-3 TENSOR MODEL AND ITS SYMMETRIES}

The $O\left(N_{1}\right) \times O\left(N_{2}\right) \times O\left(N_{3}\right)$ tensor model is specified by the action

$$
S=\int d t\left(\frac{i}{2} \psi^{a b c} \partial_{t} \psi^{a b c}-H\right),
$$

where $H$ is given in (1.2). Sometimes it will be convenient to use capital letters $A, B, \ldots$ to denote the multi-index, i.e.
$A=(a, b, c)$. It is easy to see that the Hamiltonian (1.2) has a traceless spectrum ${ }^{2}$ :

$$
\sum_{i} d_{i} E_{i}=0, \quad \sum_{i} d_{i}=2^{\left[N_{1} N_{2} N_{3} / 2\right]},
$$

where $d_{i}$ is the degeneracy of eigenvalue $E_{i}$.

We can make some general restrictions on the possible values of the energies. Operators $\psi$ obeying the anticommutation relation (1.1) may be represented as the Majorana $\gamma$-matrices in $N_{1} N_{2} N_{3}$-dimensional Euclidean space. They have entries which, in our conventions, are integers divided by $\sqrt{2}$. As a result, the Hamiltonian is an integer matrix times $g / 16$. It is a well-known mathematical fact that such matrices cannot have rational eigenvalues. Therefore, in units of $g / 16$, the energy eigenvalues have to be either integer or irrational numbers. The explicit results we will find are in agreement with this.

The discrete symmetries of the theory depend on whether some of the ranks are equal. In a $O\left(N_{1}\right) \times$ $O(N)^{2}$ theory, $N_{1} \neq N$, we may study interchange of the two $O(N)$ groups, which acts as $\psi^{a b c} \rightarrow \psi^{a c b}$. The invariant operators can be divided into even or odd under the interchange. The Hamiltonian (1.2) is odd [34], which implies that the energy spectrum is symmetric under $E \rightarrow-E$.

Let us construct the operator which implements the interchange $\psi^{a b c} \rightarrow \psi^{a c b}$ :

$$
P_{23}=2^{N^{2}\left(N_{1}+1\right) / 2} \prod_{a, b=c} \psi^{a b c} \prod_{a, b>c}\left(\frac{\psi^{a b c}+\psi^{a c b}}{\sqrt{2}}\right) .
$$

This operator has the following properties

$$
\begin{aligned}
P_{23}^{\dagger} P_{23} & =1, \quad P_{23}^{\dagger}= \pm P_{23}, \\
P_{b c}^{\dagger} \psi^{a b c} P_{b c} & =(-1)^{N^{2}\left(N_{1}+1\right) / 2+1} \psi^{a c b} .
\end{aligned}
$$

Due to the last relation one can check

$$
\begin{aligned}
P_{b c}^{\dagger} H P_{b c} & =P_{b c}^{\dagger}\left(\frac{g}{4} \psi^{a b c} \psi^{a b^{\prime} c^{\prime}} \psi^{a^{\prime} b c^{\prime}} \psi^{a^{\prime} b^{\prime} c}-\frac{g}{16} N_{1} N_{2} N_{3}\left(N_{1}-N_{2}+N_{3}\right)\right) P_{b c} \\
& =\frac{g}{4} \psi^{a c b} \psi^{a c^{\prime} b^{\prime}} \psi^{a^{\prime} c^{\prime} b} \psi^{a^{\prime} c b^{\prime}}-\frac{g}{16} N_{1} N_{2} N_{3}\left(N_{1}-N_{2}+N_{3}\right) \\
& =-\frac{g}{4} \psi^{a b c} \psi^{a b^{\prime} c^{\prime}} \psi^{a^{\prime} b c^{\prime}} \psi^{a^{\prime} b^{\prime} c}+\frac{g}{16} N_{1} N_{2} N_{3}\left(N_{1}-N_{2}+N_{3}\right)=-H,
\end{aligned}
$$

where we have renamed the repeated indices in the second line and used the anticommutation relations (1.1) in the third line. Let us consider any state that is an eigenvector of the $P_{23}$, it exists because $P_{23}$ is either Hermitian or anti-Hermitian

$$
P_{23}|\lambda\rangle=\lambda|\lambda\rangle, \quad 1=\langle\lambda \mid \lambda\rangle=\left\langle\lambda\left|P_{23}^{\dagger} P_{23}\right| \lambda\right\rangle=|\lambda|^{2}\langle\lambda \mid \lambda\rangle=|\lambda|^{2} .
$$

\footnotetext{
${ }^{2}$ One can easily compute $\operatorname{tr}\left(\psi^{a b c} \psi^{a b^{\prime} c^{\prime}} \psi^{a^{\prime} b c^{\prime}} \psi^{a^{\prime} b^{\prime} c}\right)=\frac{1}{4} N_{1} N_{2} N_{3}\left(N_{1}-N_{2}+N_{3}\right)$ working with $\psi^{a b c}$ as with a set of gamma matrices.
} 
The energy of such state is equal to zero. Indeed,

$$
\begin{aligned}
E & =\langle\lambda|H| \lambda\rangle=-\left\langle\lambda\left|P_{b c}^{\dagger} H P_{b c}\right| \lambda\right\rangle \\
& =-|\lambda|^{2}\langle\lambda|H| \lambda\rangle=-E, \quad E=0
\end{aligned}
$$

Let us now discuss the case when all three ranks are equal and we have $O(N)^{3}$ symmetry. Then the invariant operators form irreducible representations of the group $S_{3}$ which interchanges the $3 O(N)$ groups. The Hamiltonian is in the sign representation of degree 1: it is invariant under the even permutations and changes sign under the odd ones. Therefore, the symmetry of the Hamiltonian is the alternating group $A_{3}$, which is isomorphic to $Z_{3}$. Two types of eigenstates are possible: the ones that are invariant under $Z_{3}$ are nondegenerate, but the ones which pick up a phase $e^{ \pm 2 \pi i / 3}$ are doubly degenerate.

The $S O\left(N_{i}\right)$ symmetry charges are

$$
\begin{aligned}
Q_{1}^{a a^{\prime}} & =\frac{i}{2}\left[\psi^{a b c}, \psi^{a^{\prime} b c}\right], \\
Q_{2}^{b b^{\prime}} & =\frac{i}{2}\left[\psi^{a b c}, \psi^{a b^{\prime} c}\right], \\
Q_{3}^{c c^{\prime}} & =\frac{i}{2}\left[\psi^{a b c}, \psi^{a b c^{\prime}}\right] .
\end{aligned}
$$

In addition, each $O\left(N_{i}\right)$ group contains $Z_{2}$ parity symmetries which are axis reflections. Inside $O\left(N_{1}\right)$ there are parity symmetries $P^{a^{\prime}}$ : for a given $a^{\prime}, P^{a^{\prime}}$ sends $\psi^{a^{\prime} b c} \rightarrow$ $-\psi^{a^{\prime} b c}$ for all $b, c$ and leaves all $\psi^{a b c}, a \neq a^{\prime}$ invariant. It is not hard to see that the corresponding charges are

$$
P^{a^{\prime}}=2^{N_{2} N_{3}} \prod_{b c} \psi^{a^{\prime} b c}
$$

One can indeed check that

$$
\left(P^{a^{\prime}}\right)^{\dagger} \psi^{a b c} P^{a^{\prime}}=(-1)^{\delta_{a, a^{\prime}}+N_{2} N_{3}} \psi^{a b c} .
$$

Similarly, there are $Z_{2}$ charges inside $O\left(N_{2}\right)$ and $O\left(N_{3}\right)$. A product of two different parity symmetries within the same $O\left(N_{i}\right)$ group is a $S O\left(N_{i}\right)$ rotation. Therefore, it is enough to consider one such $Z_{2}$ parity symmetry within each group and $O\left(N_{i}\right) \sim S O\left(N_{i}\right) \times Z_{2}$.

The antiunitary time reversal symmetry $\mathcal{T}$ is a general feature of systems of Majorana fermions; it commutes with them and, therefore, with the Hamiltonian (1.2):

$$
\mathcal{T}^{-1} \psi_{a b c} \mathcal{T}=\psi_{a b c} .
$$

The action of $\mathcal{T}$ on the eigenstates depends on the total number of the Majorana fermions $N_{1} N_{2} N_{3}$ and is well known in the theory of topological insulators and superconductors [50]. If the total number of fermions is divisible by 8 , the operator $\mathcal{T}$ acts trivially, so the ground state may be nondegenerate. Otherwise $\mathcal{T}$ acts nontrivially and one finds that the ground state must be degenerate.

\section{ENERGY BOUNDS FOR THE $O\left(N_{1}\right) \times O\left(N_{2}\right) \times O\left(N_{3}\right)$ MODEL}

Since the Hilbert space of the model is finite dimensional, it is interesting to put an upper bound on the absolute value of the energy eigenvalues in each representation of the symmetry group. In this section we address this question in two different ways. We first derive a basic linear relation between the Hamiltonian, a quadratic Casimir operator, and a square of a Hermitian operator which is positive definite. This gives bounds which are useful for the representations where the quadratic Casimir of one of the orthogonal groups is near its maximum allowed value. We also find that the bounds are exactly saturated for $N_{3}=2$, but are not stringent when equal ranks become large. Then in Sec. III B we derive more refined bounds which are more stringent in the large $N$ limit and give the expected scaling of the ground state energy. Furthermore, we derive a finite multiplicative factor which corrects the refined bound and allows us to deduce the ground state energy in the large $N$ limit.

\section{A. Basic bounds}

To derive an energy bound we introduce the Hermitian tensor

$$
\begin{aligned}
A^{b c, b^{\prime} c^{\prime}} & =\frac{i}{2}\left[\psi^{a b c}, \psi^{a b^{\prime} c^{\prime}}\right]=i \psi^{a b c} \psi^{a b^{\prime} c^{\prime}}-i \frac{N_{1}}{2} \delta^{b b^{\prime}} \delta^{c c^{\prime}} \\
\left(A^{b c, b^{\prime} c^{\prime}}\right)^{\dagger} & =-i \psi^{a b^{\prime} c^{\prime}} \psi^{a b c}+i \frac{N_{1}}{2} \delta^{b b^{\prime}} \delta^{c c^{\prime}} \\
& =i \psi^{a b c} \psi^{a b^{\prime} c^{\prime}}-i \frac{N_{1}}{2} \delta^{b b^{\prime}} \delta^{c c^{\prime}}=A^{b c, b^{\prime} c^{\prime}}
\end{aligned}
$$

If we think of $b c$ as a combined index which takes $N_{2} N_{3}$ values, then $A^{b c, b^{\prime} c^{\prime}}$ are the generators of the transformations in $O\left(N_{2} N_{3}\right) \supset O\left(N_{2}\right) \times O\left(N_{3}\right)$. The quadratic Casimir of $O\left(N_{2} N_{3}\right) \supset O\left(N_{2}\right) \times O\left(N_{3}\right)$,

$$
C_{2}^{O\left(N_{2} N_{3}\right)}=\frac{1}{2} A^{b c, b^{\prime} c^{\prime}} A^{b c, b^{\prime} c^{\prime}},
$$

and the quadratic Casimir of the $O\left(N_{1}\right)$ symmetry,

$$
C_{2}^{O\left(N_{1}\right)}=\frac{1}{2} Q_{1}^{a a^{\prime}} Q_{1}^{a a^{\prime}}
$$

are related by

$C_{2}^{O\left(N_{2} N_{3}\right)}+C_{2}^{O\left(N_{1}\right)}=\frac{N_{1} N_{2} N_{3}}{8}\left(N_{1}+N_{2} N_{3}-2\right)$.

Therefore, for the states which appear in the model, we find the upper bound:

$$
C_{2}^{O\left(N_{1}\right)} \leq \frac{1}{8} N_{1} N_{2} N_{3}\left(N_{1}+N_{2} N_{3}-2\right) .
$$


This bound is saturated only if $C_{2}^{O\left(N_{2} N_{3}\right)}=0$ so that the state is invariant under $\mathrm{SO}\left(\mathrm{N}_{2} \mathrm{~N}_{3}\right)$.

The Hamiltonian may be written as

$H=-\frac{g}{4} A^{b c, b^{\prime} c^{\prime}} A^{b c^{\prime}, b^{\prime} c}+\frac{g}{16} N_{1} N_{2} N_{3}\left(N_{2}-N_{3}\right)$.

Now we note the inequality

$$
C_{2}^{O\left(N_{2} N_{3}\right)} \pm \frac{1}{2} A^{b c, b^{\prime} c^{\prime}} A^{b c^{\prime}, b^{\prime} c}=\frac{1}{4}\left(A^{b c, b^{\prime} c^{\prime}} \pm A^{b c^{\prime}, b^{\prime} c}\right)^{2} \geq 0
$$

Combining this with (3.4) we get

$\frac{2}{g} H\left\{\begin{array}{l}\leq \frac{1}{8} N_{1} N_{2} N_{3}\left(N_{1}+N_{2}-N_{3}+N_{2} N_{3}-2\right)-C_{2}^{O\left(N_{1}\right)}, \\ \geq C_{2}^{O\left(N_{1}\right)}-\frac{1}{8} N_{1} N_{2} N_{3}\left(N_{1}-N_{2}+N_{3}+N_{2} N_{3}-2\right) .\end{array}\right.$

In an analogous fashion we can also derive the bounds in terms of $C_{2}$ :

$$
\frac{2}{g} H\left\{\begin{array}{l}
\leq \frac{1}{8} N_{1} N_{2} N_{3}\left(N_{2}+N_{3}-N_{1}+N_{1} N_{3}-2\right)-C_{2}^{O\left(N_{2}\right)}, \\
\geq C_{2}^{O\left(N_{2}\right)}-\frac{1}{8} N_{1} N_{2} N_{3}\left(N_{2}-N_{3}+N_{1}+N_{1} N_{3}-2\right)
\end{array}\right.
$$

and similarly in terms of $C_{2}^{O\left(N_{3}\right)}$.

An interesting special case, which we will consider in Sec. VI, is $N_{3}=2$ where we find a complex $N_{1} \times N_{2}$ matrix model. For the singlet states where $C_{2}^{O\left(N_{1}\right)}=$ $C_{2}^{O\left(N_{2}\right)}=0$ the most stringent bound we get from (3.8) and (3.9) is

$$
|H| \leq \frac{g}{8} N_{1} N_{2}\left(N_{1}+N_{2}\right) .
$$

In Sec. VI we will show that these bounds are saturated by the exact solution for even $N_{1}, N_{2}$. For $N_{1}=N_{2}=N$ we have a $N \times N$ matrix quantum mechanics which possesses a 't Hooft large $N$ limit where $g N=\lambda$ is held fixed. In this limit, the ground state energy is $E_{0}=-\frac{\lambda}{4} N^{2}$, which has the expected scaling with $N$ for a matrix model.

More generally, if at least one of the ranks is even (we will call it $N_{3}$ ), we may introduce the operators [44]

$$
\begin{aligned}
\bar{c}_{a b k} & =\frac{1}{\sqrt{2}}\left(\psi^{a b(2 k-1)}+i \psi^{a b(2 k)}\right), \\
c_{a b k} & =\frac{1}{\sqrt{2}}\left(\psi^{a b(2 k-1)}-i \psi^{a b(2 k)}\right), \\
\left\{c_{a b k}, c_{a^{\prime} b^{\prime} k^{\prime}}\right\} & =\left\{\bar{c}_{a b k}, \bar{c}_{a^{\prime} b^{\prime} k^{\prime}}\right\}=0, \\
\left\{\bar{c}_{a b k}, c_{a^{\prime} b^{\prime} k^{\prime}}\right\} & =\delta_{a a^{\prime}} \delta_{b b^{\prime}} \delta_{k k^{\prime}},
\end{aligned}
$$

where $a=1,2, \ldots, N_{1}, b=1,2 \ldots, N_{2}$ and $k=1, \ldots, \frac{N_{3}}{2}$. In this basis the $O\left(N_{1}\right) \times O\left(N_{2}\right) \times U\left(N_{3} / 2\right)$ symmetry is manifest. The Hamiltonian becomes [44]

$$
\begin{aligned}
H= & \frac{g}{2}\left(\bar{c}_{a b k} \bar{c}_{a b^{\prime} k^{\prime}} c_{a^{\prime} b k^{\prime}} c_{a^{\prime} b^{\prime} k}-\bar{c}_{a b k} \bar{c}_{a^{\prime} b k^{\prime}} c_{a b^{\prime} k^{\prime}} c_{a^{\prime} b^{\prime} k}\right) \\
& +\frac{g}{16} N_{1} N_{2} N_{3}\left(N_{2}-N_{1}\right) .
\end{aligned}
$$

It is invariant under the charge conjugation symmetry which interchanges $c_{a b k}$ with $\bar{c}_{a b k}$.

For any even $N_{3}$, using the basis (3.11) we define the oscillator vacuum state by the condition $c_{a b k}|0\rangle=0$. Since this condition is invariant under $O\left(N_{1} N_{2}\right)$, so is $|0\rangle$. Furthermore, all the states that are created by operators that are $O\left(N_{1} N_{2}\right)$ invariant are also $O\left(N_{1} N_{2}\right)$ invariant and have energy $\frac{g}{16} N_{1} N_{2} N_{3}\left(N_{2}-N_{1}\right)$. The number of such states is estimated to be the dimension of the maximal representation for the $O\left(N_{3}\right)$ group $\operatorname{dim}_{\max } \sim\left(N_{1} N_{2}\right)^{N_{3}^{2} / 8}$ (see Appendix A for details). The relation (3.4) also simplifies the search for the singlets. For example, we can first forget about the group nature of the third index in the approach of [44] and impose the vanishing of the Casimir of the third group afterwards. By studying the charges under $U(1) \in U\left(N_{3} / 2\right)$ we find that the singlet states must have $N_{1} N_{2} N_{3} / 4$ creation operators acting on $|0\rangle$.

Specifying the bound (3.8) to the equal ranks $N_{1}=$ $N_{2}=N_{3}=N$, we find

$$
\begin{aligned}
& C_{2}^{O(N)_{1}}-\frac{1}{8} N^{3}(N+2)(N-1) \\
& \quad \leq \frac{2}{g} H \leq \frac{1}{8} N^{3}(N+2)(N-1)-C_{2}^{O(N)_{1} .}
\end{aligned}
$$

When the bound (3.5) is saturated, the corresponding state must have zero energy. This shows that all the states invariant under $O\left(N^{2}\right) \supset O(N)_{2} \times O(N)_{3}$ have $E=0$.

For the singlet states (3.13) gives

$$
\frac{4}{g}|H| \leq \frac{1}{4} N^{3}(N+2)(N-1) .
$$

For $N=2$, exact diagonalization gives that the ground states is a singlet with energy $E_{0}=-2 g$; this saturates the bound (3.14). For $N=3$, exact diagonalization gives a ground state with energy $-\frac{5}{4} \sqrt{41} g \approx-8.0039 g$, which is in the $(2,2,2)$ representation of $O(3)^{3}$. Since for the 2 of $S O(3), C_{1}=3 / 4$, the bound (3.13) is $E_{0} \geq-\frac{33}{2} g$. This is satisfied and is far from being saturated.

In the large $N$ limit, $J=g N^{3 / 2}$ is held fixed. Thus, we obtain a bound on the lowest singlet energy $E_{0}$, which is $E_{0} \geq-c J N^{7 / 2}$, where $c$ is a positive constant. Since we expect the ground state energy to be of order $N^{3}$, this bound is not very informative at large $N$. A better bound at large $N$ will be derived in the next section.

\section{B. Refined bounds}

In this section we present another approach to deriving energy bounds for the $O\left(N_{1}\right) \times O\left(N_{2}\right) \times O\left(N_{3}\right)$ invariant 
states, which gives a more stringent bound in the large $N$ limit than the ones in the previous section.

Consider an arbitrary singlet density matrix $\rho$; this means a density matrix invariant under the $O\left(N_{1}\right) \times O\left(N_{2}\right) \times$ $O\left(N_{3}\right)$ rotations. For example, it can be $\rho_{s}=|s\rangle\langle s|$, where $|s\rangle$ is an singlet state, or if we have some representation $\mathcal{R}$ of the $O\left(N_{1}\right) \times O\left(N_{2}\right) \times O\left(N_{3}\right)$ with an orthonormal basis $\left|e_{i}\right\rangle, i=1 \ldots \operatorname{dim} \mathcal{R}$ we can define the projector on this subspace of the Hilbert space

$\rho_{\mathcal{R}}=\frac{1}{\operatorname{dim} \mathcal{R}} \sum_{i=1}^{\operatorname{dim} \mathcal{R}}\left|e_{i}\right\rangle\left\langle e_{i}\right|, \quad \rho_{R}=1, \quad \rho_{\mathcal{R}}^{2}=\frac{1}{\operatorname{dim} \mathcal{R}} \rho_{\mathcal{R}}$.

It is easy to see, that this density matrix is invariant under rotations $O^{T} \rho_{\mathcal{R}} O=\rho_{\mathcal{R}}$ for any $O \in O\left(N_{1}\right) \times O\left(N_{2}\right) \times$ $O\left(N_{3}\right)$. We can calculate the mean value of the energy for this density matrix as

$$
\begin{aligned}
E=\operatorname{tr}\left[\rho_{\mathcal{R}} H\right]= & \frac{g}{4} \operatorname{tr}\left[\rho \psi^{a b c} \psi^{a b^{\prime} c^{\prime}} \psi^{a^{\prime} b c^{\prime}} \psi^{a^{\prime} b^{\prime} c}\right] \\
& -\frac{g}{16} N_{1} N_{2} N_{3}\left(N_{1}-N_{2}+N_{3}\right) .
\end{aligned}
$$

For a fixed $a, b, c$ we can act by the rotation matrices (that act trivially on the singlet density matrix $\rho_{s}$ ) and make the interchangement $a \leftrightarrow 1, b \leftrightarrow 1, c \leftrightarrow 1$. This argument gives us that

$E=\frac{g}{4} N_{1} N_{2} N_{3} \operatorname{tr}\left[\rho_{\mathcal{R}} h\right]-\frac{g}{16} N_{1} N_{2} N_{3}\left(N_{1}-N_{2}+N_{3}\right)$,

$h=\psi^{111} \psi^{1 b^{\prime} c^{\prime}} \psi^{a^{\prime} 1 c^{\prime}} \psi^{a^{\prime} b^{\prime} 1}$,

where we sum over the repeated indexes. From now on we consider the density matrix to be of the form (3.15). Now we can estimate the trace in the formula (3.17). With the use of Cauchy-Schwarz inequality, we have

$$
\begin{aligned}
\operatorname{tr}\left[\rho_{\mathcal{R}} h\right]^{2} & \leq \operatorname{tr}\left[\rho_{\mathcal{R}} h^{\dagger} h\right] \\
& =\frac{1}{2} \operatorname{tr}\left[\rho_{\mathcal{R}} \psi^{a b 1} \psi^{a 1 c} \psi^{1 b c} \psi^{1 b^{\prime} c^{\prime}} \psi^{a^{\prime} 1 c^{\prime}} \psi^{a^{\prime} b^{\prime} 1}\right] .
\end{aligned}
$$

Because the density matrix $\rho_{\mathcal{R}}$ is a singlet we can rotate indexes back to get

$$
\begin{aligned}
\operatorname{tr}\left[\rho_{\mathcal{R}} h\right]^{2} & \leq \frac{1}{2 N_{1} N_{2} N_{3}} \operatorname{tr}\left[\rho_{\mathcal{R}} q_{a b c}^{\dagger} q_{a b c}\right], \\
q_{a b c} & =\psi^{a b^{\prime} c^{\prime}} \psi^{a^{\prime} b c^{\prime}} \psi^{a^{\prime} b^{\prime} c} .
\end{aligned}
$$

We can express it in the following way

$$
\begin{aligned}
& \left(\operatorname{tr}\left[\rho_{\mathcal{R}} h\right]-\frac{1}{4}\left(N_{1}-N_{2}+N_{3}\right)\right)^{2} \\
& \quad \leq \frac{1}{2 N_{1} N_{2} N_{3}} \operatorname{tr}\left[\rho_{\mathcal{R}} q_{a b c}^{2}\right]+\frac{1}{16}\left(N_{1}-N_{2}+N_{3}\right)^{2}
\end{aligned}
$$

The square of the operator $q_{a b c}$ can be expressed as a sum of Casimir operators due to the virtue of the anticommutation relations. That gives us the bound on the energies of states in representation $\mathcal{R}$ :

$$
\begin{aligned}
\left|E_{\mathcal{R}}\right| \leq & \frac{g}{16} N_{1} N_{2} N_{3}\left(N_{1} N_{2} N_{3}+N_{1}^{2}+N_{2}^{2}+N_{3}^{2}\right. \\
& \left.-4-\frac{8}{N_{1} N_{2} N_{3}} \sum_{i=1}^{3}\left(N_{i}+2\right) C_{i}^{\mathcal{R}}\right)^{1 / 2},
\end{aligned}
$$

where $C_{i}^{\mathcal{R}}$ is the value of Casimir operator in the representation $\mathcal{R}$. For the singlet states this gives

$$
|E| \leq \frac{g}{16} N_{1} N_{2} N_{3}\left(N_{1} N_{2} N_{3}+N_{1}^{2}+N_{2}^{2}+N_{3}^{2}-4\right)^{1 / 2} .
$$

Since $C_{i} \geq 0$ this bound applies to all energies. Let us note that for $N_{3}=2$ the square root may be taken explicitly:

$$
|E|_{N_{3}=2} \leq \frac{g}{8} N_{1} N_{2}\left(N_{1}+N_{2}\right),
$$

which is identical to the earlier result (3.10). In Sec. VI we will show that this is saturated when $N_{1}, N_{2}$ are even and the ground state is a singlet.

For the case when $N_{1}=N_{2}=N_{3}=N$ and $N>2$ the bound (3.22) is more stringent than the earlier bound (3.14):

$$
|E| \leq E_{\text {bound }}=\frac{g}{16} N^{3}(N+2) \sqrt{N-1}
$$

In the large $N$ limit, $E_{\text {bound }} \rightarrow J N^{3} / 16$, which is the expected behavior of the ground state energy; in the melonic limit it scales as $N^{3}$. We may expand (3.21) for large $N$ to find

$$
\begin{aligned}
\left|E_{\mathcal{R}}\right| \leq & \frac{g}{16} N^{3}(N+2) \sqrt{N-1} \\
& \times\left(1-\frac{4}{(N+2)(N-1) N^{3}} \sum_{i=1}^{3} C_{i}^{\mathcal{R}}+\ldots\right) .
\end{aligned}
$$

The discussion of the splittings between nonsinglet and singlet states in Sec. IV will be in agreement with the scaling of the second term.

We can try to estimate how close the singlet ground state $|\mathrm{vac}\rangle$ comes to the bound (3.24) by using the exact propagator $G(t)=\left\langle T \psi^{a b c}(t) \psi^{\operatorname{def}}(0)\right\rangle$ in the large $N$ limit. To do it let us consider the two states

$$
|1\rangle=\psi^{111}|\mathrm{vac}\rangle, \quad|2\rangle=\partial_{t} \psi^{111}|\mathrm{vac}\rangle
$$

where we have introduced $\partial_{t} \psi_{a b c}=i\left[H, \psi^{a b c}\right]$. We can introduce the angle $\theta$ between these states 


$$
\begin{aligned}
\cos ^{2} \theta=\frac{|\langle 1 \mid 2\rangle|^{2}}{\langle 1 \mid 1\rangle\langle 2 \mid 2\rangle} & =2 \frac{\left|\left\langle\operatorname{vac}\left|\psi^{111} \partial_{t} \psi^{111}\right| \mathrm{vac}\right\rangle\right|^{2}}{\left\langle\operatorname{vac}\left|\left(\partial_{t} \psi^{111}\right)^{2}\right| \mathrm{vac}\right\rangle} \\
& =2 \frac{\left|\left\langle\operatorname{vac}\left|\psi^{a b c} \partial_{t} \psi^{a b c}\right| \mathrm{vac}\right\rangle\right|^{2}}{N_{1} N_{2} N_{3}\left\langle\operatorname{vac}\left|\left(\partial_{t} \psi^{a b c}\right)^{2}\right| \mathrm{vac}\right\rangle},
\end{aligned}
$$

where we have rotated back the indexes back by using the fact that the $|v a c\rangle$ is a singlet state. One can notice $H=i \psi^{a b c} \partial_{t} \psi^{a b c}$, while $\left\langle\operatorname{vac}\left|\left(\partial_{t} \psi^{a b c}\right)^{2}\right| \operatorname{vac}\right\rangle$ is just equal to the bound (3.22), then

$$
\cos ^{2} \theta=\frac{E_{0}^{2}}{E_{\text {bound }}^{2}},
$$

where $E_{\text {bound }}$ is the bound on the singlet ground state energy (3.24). The other way to estimate this angle $\theta$ can be done in the following way. We shift the Hamiltonian, such that the ground state has the zero energy $\left(H-E_{0}\right)|\mathrm{vac}\rangle=$ 0 and calculate the expectation value for the energy for the state $|1\rangle$ :

$$
\begin{aligned}
\langle E\rangle_{1}=\frac{\left\langle 1\left|\left(H-E_{0}\right)\right| 1\right\rangle}{\langle 1 \mid 1\rangle} & =2\left\langle\operatorname{vac}\left|\psi^{111}\left(H-E_{0}\right) \psi^{111}\right| \mathrm{vac}\right\rangle \\
& =2 i\left\langle\operatorname{vac}\left|\psi^{111} \partial_{t} \psi^{111}\right| \mathrm{vac}\right\rangle
\end{aligned}
$$

at the same time the second moment of the energy is

$$
\begin{aligned}
\left\langle E^{2}\right\rangle_{1}=\frac{\left\langle 1\left|\left(H-E_{0}\right)^{2}\right| 1\right\rangle}{\langle 1 \mid 1\rangle} & =2\left\langle\operatorname{vac}\left|\psi^{111}\left(H-E_{0}\right)^{2} \psi^{111}\right| \mathrm{vac}\right\rangle \\
& =-2 g^{2}\left\langle\operatorname{vac}\left|\left(\partial_{t} \psi^{111}\right)^{2}\right| \mathrm{vac}\right\rangle .
\end{aligned}
$$

Where we have used the fact that $\partial_{t} \psi_{a b c}=i\left[H, \psi^{a b c}\right]$. After that we can notice that (3.27) can be rewritten as

$$
\cos ^{2} \theta=\frac{\langle E\rangle_{1}^{2}}{\left\langle E^{2}\right\rangle_{1}}
$$

If $\cos \theta=1$, it means that $\langle E\rangle_{1}^{2}=\left\langle E^{2}\right\rangle_{1}$ that can be true only if and if $\psi^{111}|\mathrm{vac}\rangle$ is an eigenstate of the Hamiltonian. It would give that the propagator is

$$
G(t)=\left\langle\psi^{a b c} e^{-i H t} \psi^{a^{\prime} b^{\prime} c^{\prime}}\right\rangle \propto \delta^{a a^{\prime}} \delta^{b b^{\prime}} \delta^{c c^{\prime}} e^{-i \Delta E|t|} .
$$

But as we know the solution for the propagator in the large $N$ limit is a conformal propagator. From this we deduce that the bound can not be saturated. Nevertheless we can estimate the angle $\cos ^{2} \theta$. Indeed, in the large $N$ limit the propagator can be calculated numerically or approximated by a conformal one. From this we can calculate the $\langle E\rangle_{1}$ and $\left\langle E^{2}\right\rangle_{1}$. We assume $t>t^{\prime}=0, a=a^{\prime}, b=b^{\prime}$, $c=c^{\prime}$ and insert the full basis $\left|E_{n}\right\rangle$ of eigenstates of the Hamiltonian in the propagator to get

$$
\begin{aligned}
\left\langle\psi_{a b c}(t) \psi_{a b c}(0)\right\rangle & =\sum_{n}\left|\left\langle\operatorname{vac}\left|\psi_{a b c}\right| E_{n}\right\rangle\right|^{2} e^{-i\left(E_{n}-E_{0}\right) t} \\
& =\int_{0}^{\infty} d E \rho(E) e^{-i E t}, \\
\text { where } \rho(E) & =\sum_{n}\left|\left\langle\operatorname{vac}\left|\psi_{a b c}\right| E_{n}\right\rangle\right|^{2} \delta\left(E-E_{n}+E_{0}\right) .
\end{aligned}
$$

The function $\rho(E)$ is known as a structure factor. From this we can calculate

$$
\begin{aligned}
\langle E\rangle_{1} & =\int_{0}^{\infty} d E E \rho(E), \\
\left\langle E^{2}\right\rangle_{1} & =\int_{0}^{\infty} d E E^{2} \rho(E), \\
\cos ^{2} \theta & =\frac{\langle E\rangle_{1}^{2}}{\left\langle E^{2}\right\rangle_{1}}
\end{aligned}
$$

One can use conformal propagator to estimate this angle, which gives $\cos \theta \approx 0.745$, while the numerical calculation [22] gives $\cos \theta=0.6608$. From this and the formula (3.28) we get the ground state energy in the large $N$ limit:

$E_{0} \rightarrow-\cos \theta E_{\text {bound }}=-\cos \theta \frac{J N^{3}}{16} \approx-0.041 J N^{3}$.

This answer is close to the numerical result for the ground state energy in the SYK model [39]: $E_{0} \approx-0.04 J N_{\mathrm{SYK}}$. One can make analogous calculations for the other representations. It gives us in the large $N$ limit the following formula for the gap to the lowest state in a representation $\mathcal{R}$ :

$$
E-E_{0}=\frac{J \cos \theta}{4 N^{2}} \sum_{i=1}^{3} C_{i}^{\mathcal{R}}
$$

\section{SIGMA MODEL AND ENERGY GAPS}

In the large $N$ limit the model (2.1) is dominated by melonic diagrams. This allows one to write down a closed system of Schwinger-Dyson equations for the Green function $G_{a^{\prime} b^{\prime} c^{\prime}}^{a b c}\left(t_{1}-t_{2}\right)=\left\langle T \psi^{a b c}\left(t_{1}\right) \psi^{a^{\prime} b^{\prime} c^{\prime}}\left(t_{2}\right)\right\rangle$ and selfenergy $\Sigma_{a^{\prime} b^{\prime} c^{\prime}}^{a b c}$ and the bare Green function $G_{a^{\prime} b^{\prime} c^{\prime}, 0}^{a b c}(\omega)=$ $i \delta_{a^{\prime}}^{a} \delta_{b^{\prime}}^{b} \delta_{c^{\prime}}^{c} / \omega$

$$
\begin{aligned}
\left(G_{a^{\prime} b^{\prime} c^{\prime}}^{a b c}(\omega)\right)^{-1} & =\left(G_{a^{\prime} b^{\prime} c^{\prime}, 0}^{a b c}(\omega)\right)^{-1}-\Sigma_{a^{\prime} b^{\prime} c^{\prime}}^{a b c}(\omega), \\
\sum_{a^{\prime} b^{\prime} c^{\prime}}^{a b c}(t) & =g^{2} G_{a^{\prime} \beta^{\prime} \gamma^{\prime}}^{a \beta \gamma}(t) G_{\alpha^{\prime} b^{\prime} \gamma^{\prime}}^{\alpha b \gamma}(t) G_{\alpha^{\prime} \beta^{\prime} c^{\prime}}^{\alpha \beta c}(t) .
\end{aligned}
$$

For simplicity we shall introduce the multi-index $A=(a, b, c)$. We can look for a solution in the diagonal form $G^{A B}=G(t) \delta^{A B}$ and $\Sigma^{A B}=\Sigma(t) \delta^{A B}$. Then we have the following set of equations: 


$$
G^{-1}(\omega)=-i \omega-\Sigma(\omega), \quad \Sigma(t)=J^{2} G^{3}(t) .
$$

These equations exactly coincide with the SchwingerDyson equations of the SYK model and have a conformal solution.

It was argued in [35] that the system of equations (4.1) can be obtained from the effective action ${ }^{3}$ :

$$
\begin{aligned}
S_{\text {eff }}= & -\log \operatorname{Pf}\left(\delta_{A B} \partial_{t}+\Sigma_{A B}\right) \\
& +\int d t_{1} d t_{2}\left(-\Sigma_{A B}\left(t_{1}-t_{2}\right) G^{A B}\left(t_{2}-t_{1}\right)\right. \\
& \left.-\frac{g^{2}}{4} G^{4}\left(t_{1}-t_{2}\right)\right)
\end{aligned}
$$

This action was recently derived from two-particle irreducible diagrams in [33].

In the strong coupling limit $J \rightarrow \infty$ one can neglect the bare Green function. Then, as first noticed in [35], the global symmetry $O(N)^{3}$ is promoted to the gauged symmetry $O(N)^{3}$. Indeed, if we neglect $G_{0}^{A B}(\omega)$ in (4.1) then it is easy to see that we can generate a series of solutions by doing a gauge transformation:

$$
\begin{aligned}
& G_{A B}\left(t_{1}-t_{2}\right) \rightarrow\left(O_{A A^{\prime}}\left(t_{1}\right)\right)^{T} G_{A^{\prime} B^{\prime}}\left(t_{1}-t_{2}\right) O_{B B^{\prime}}\left(t_{2}\right) \\
& \Sigma_{A B}\left(t_{1}-t_{2}\right) \rightarrow\left(O_{A A^{\prime}}\left(t_{1}\right)\right)^{T} \Sigma_{A^{\prime} B^{\prime}}\left(t_{1}-t_{2}\right) O_{B B^{\prime}}\left(t_{2}\right)
\end{aligned}
$$

where we introduce matrix $O$ in $O(N)^{3}$ which equals to $O_{A B}=O_{\alpha \alpha^{\prime}}^{1} O_{\beta \beta^{\prime}}^{2} O_{\gamma \gamma^{\prime}}^{3}$.

The effective action (5.17) is also invariant under these transformations if one omits the term $\partial_{t}$ in the Pfaffian. For finite $J$, the action ceases to be invariant. If we plug the gauge transformation (4.4) into the effective action (5.17), the potential does not change, while we will get a kinetic term for matrices $O^{i}$ of order $1 / J$. Indeed, for the conformal solution we have $\Sigma_{A B}=-(1 / G)_{A B}$ and we can rewrite the kinetic part of the action as

$$
\begin{aligned}
& -\log \operatorname{Pf}\left(\delta_{A B} \partial_{t}+\Sigma_{A B}\right) \\
& \quad=-\log \operatorname{Pf}\left(\delta_{A B}-\partial_{t} G_{A B}\right)-\log \operatorname{Pf}\left(\Sigma_{A B}\right) .
\end{aligned}
$$

The second term $\log \operatorname{Pf}\left(\Sigma_{A B}\right)$ is invariant under gauge transformations. Then expanding the Pfaffian in the leading order in derivatives we get

$$
\left.\frac{1}{2} \int d t \operatorname{Tr} \partial_{t} G_{A B}\left(t, t^{\prime}\right)\right|_{t^{\prime} \rightarrow t}
$$

Making the gauge transformation (4.4) of the conformal solution $G_{A B}=\delta_{A B} G$ and plugging into (4.6) we get:

\footnotetext{
${ }^{3}$ For clarity, we have omitted the indices in the $G^{4}$ term. Explicitly, this term reads as $G_{a^{\prime} \beta^{\prime} \gamma^{\prime}}^{a \beta \gamma} G_{\alpha^{\prime} b^{\prime} \gamma^{\prime}}^{\alpha b \gamma} G_{\alpha^{\prime} \beta^{\prime} c^{\prime}}^{\alpha \beta c} G_{a^{\prime} b^{\prime} c^{\prime}}^{a b c}$.
}

$$
\begin{aligned}
& \frac{1}{2} \int d t \operatorname{Tr}\left(N^{3} \partial_{t} G+N^{2} \sum_{i=1}^{3} O_{i}^{T}(t) G\left(t-t^{\prime}\right) \partial_{t} O_{i}\left(t^{\prime}\right)\right. \\
& \left.\quad+\partial_{t} O_{i}^{T}(t) G\left(t-t^{\prime}\right) O_{i}\left(t^{\prime}\right)\right)\left.\right|_{t \rightarrow t^{\prime}}
\end{aligned}
$$

Factors $N^{2}$ come from $\operatorname{Tr}\left(O_{1}^{T} O_{1}\right)=N$. Now one has to regularize the limit $t_{2} \rightarrow t_{1}$ but this does not going to affect $N^{2}$ factors. The details are worked out in $[33,51]$. The upshot is that $G\left(t-t^{\prime}\right) O_{i}\left(t^{\prime}\right)$ becomes $\partial_{t} O_{i}(t) / J$ up to a normalization constant. This leads to the sigma model action

$$
S_{\mathrm{SM}}=\frac{\mathcal{A} N^{2}}{J} \int d t \operatorname{Tr}\left(\partial_{t} O_{1}^{T} \partial_{t} O_{1}+\partial_{t} O_{2}^{T} \partial_{t} O_{2}+\partial_{t} O_{3}^{T} \partial_{t} O_{3}\right)
$$

The spectrum of such a quantum mechanical sigma model is well known: the Hamiltonian is proportional to the quadratic Casimir and the eigenstates are simply representations of $O(N)^{3}$. In our case:

$$
H_{\text {gauge }}=\frac{J}{N^{2} \mathcal{A}}\left(C_{2}\left(O_{1}(N)\right)+C_{2}\left(O_{2}(N)\right)+C_{2}\left(O_{3}(N)\right)\right) .
$$

We note that this has the same structure as the Casimir correction to the energy bound (3.25). Since for the lowest nonsinglet representations $C_{2} \sim N$, we find the energy gap between singlets and nonsinglets to be of the order $\sim J / N$.

\section{COUNTING SINGLET STATES}

Suppose we have a free fermionic system of $N$ Majorana fermions $\psi^{I}, I=1, \ldots, M$ transforming under some representation $\mathcal{R}$ of the gauge group $G$. We want to compute the number of singlet states in such a system. In order to do it, we use the following method. The Lagrangian in the Euclidean space reads as:

$$
L=\psi^{I} \partial_{t} \psi^{I}+\psi^{I} A_{I J} \psi^{J}
$$

where $A_{I J}$ is a real gauge field in the representation $\mathcal{R}$. Since Majorana fermions anticommute with each other, $A_{I J}$ must be antisymmetric $A_{I J}=-A_{J I}$. The partition function of the gauged system at the temperature $\beta$ is

$$
Z_{\text {gauged }}=\mathcal{N} \int \mathcal{D} \psi \mathcal{D} A \exp \left(-\int_{0}^{\beta} d t L\right),
$$

where we have put the fermionic system on a circle with the circumference $\beta$ with antiperiodic boundary conditions $\psi(t)=-\psi(t+\beta)$. The normalization factor $\mathcal{N}$ can be easily recovered if we study the ungauged model. The integration over $\mathcal{D} A$ gives the volume of the gauge group 
and the integral over the fermion variables will yield just the dimension of the Hilbert space because the Hamiltonian of the ungauged theory is equal to zero $H_{\text {ungauge }}=0$. In this case the total number of states is simply $2^{M / 2}$ :

$$
\begin{aligned}
Z_{\text {ungauged }} & =2^{M / 2} \int \mathcal{D} A \\
& =\mathcal{N} \int \mathcal{D} \psi \mathcal{D} A \exp \left(-\int_{0}^{\beta} d t \psi \partial_{t} \psi\right) .
\end{aligned}
$$

From now on, we will put $\beta=1$. If we fix Lorentz gauge $\partial_{t} A=0$ with $A$ in the Cartan subalgebra, the Faddeev-Popov determinant gives the Haar measure, while the path integral over Majoranna fermions gives the partition function of the system with Hamiltonian $H=-\psi^{I} A_{I J} \psi^{J}$. Therefore the (5.2) can be rewritten as

$$
Z_{\text {gauged }}=\int \mathcal{D} A \operatorname{Tr}\left(\exp \left(-\psi^{I} A_{I J} \psi^{J}\right)\right),
$$

The expression under the trace is an operator of rotations and can be interpreted as a character of the group acting in the Hilbert space of fermions. By the virtue of the representation theory we know that the integral of the character over a group is equal to the number of the trivial representations, i.e. the number of the singlet states. Therefore, $Z_{\text {gauged }}$ equals the number of singlet states. If we insert in (5.2) a Wilson line in some representation $\mathcal{R}^{\prime}$, it gives the character of this representation:

$$
\begin{aligned}
& \left\langle\operatorname{Tr}_{\mathcal{R}^{\prime}} \exp (\oint A d t)\right\rangle \\
& \quad=\text { No. of states in the representation } \mathcal{R}^{\prime} .
\end{aligned}
$$

One can compute the partition function because the integral over $\psi$ in both (5.2) and (5.3) is Gaussian; therefore, the problem boils down to computing the Pfaffian:

$$
Z_{\text {gauged }}=2^{M / 2} \int \mathcal{D} A \frac{\operatorname{Pf}\left(\partial_{t}+A\right)}{\operatorname{Pf}\left(\partial_{t}\right)} .
$$

As discussed above, we fix $A$ to be a constant matrix in the Cartan subalgebra. The Faddeev-Popov determinant then yields the normalized Haar measure $d \lambda_{G}^{N}$ on the gauge group $G$ [52]:

$$
\int_{G} d \lambda_{G}^{N}=1
$$

Also, since $A$ is antisymmetric, the eigenvalues of $A$ are pairs of pure-imaginary numbers $i \lambda_{a},-i \lambda_{a}, a=1, \ldots,\lfloor N / 2\rfloor$. The ratio of the Pfaffians is

$$
\frac{\operatorname{Pf}\left(\partial_{t}+A\right)}{\operatorname{Pf}\left(\partial_{t}\right)}=\prod_{a=1}^{M / 2} \cos \left(\lambda_{a} / 2\right)
$$

There are different ways to derive this formula. One is to compute the ratio of determinants:

$$
\begin{aligned}
\frac{\operatorname{Det}\left(\partial_{t}+A\right)}{\operatorname{Det}\left(\partial_{t}\right)} & =\prod_{a=1}^{M / 2} \prod_{n=-\infty}^{\infty} \frac{\left(2 \pi i\left(n+\frac{1}{2}\right)+i \lambda_{a}\right)\left(2 \pi i\left(n+\frac{1}{2}\right)-i \lambda_{a}\right)}{\left(2 \pi i\left(n+\frac{1}{2}\right)\right)^{2}} \\
& =\prod_{a=1}^{M / 2} \cos \left(\lambda_{a} / 2\right)^{2}
\end{aligned}
$$

After that we note that if we go to the Fourier space, both $\partial_{t}$ and $A$ are real antisymmetric matrices, so the ratio of Pfaffians must be a real smooth function of $\lambda_{a}$. Therefore, taking the square root of Eq. (5.9) we get Eq. (5.8). Alternatively, we can calculate the Pfaffian of $\partial_{t}+A$ in Fourier space. The result is the following formula:

$$
\text { No. of singlet states }=\int d \lambda_{G}^{N} \prod_{a=1}^{M / 2} 2 \cos \left(\lambda_{a} / 2\right),
$$

where we have got the normalization by studying the ungauged theory (5.3).

Let us apply this approach to the case when Majorana fermions live in the fundamental representation of several orthogonal groups. It is important to distinguish between $S O(2 n)$ and $S O(2 n+1)$. The Cartan subalgebra in the $S O(2 n)$ algebra consists of the block diagonal matrices with $2 \times 2$ blocks

$$
\left(\begin{array}{cc}
0 & x_{i} \\
-x_{i} & 0
\end{array}\right)
$$

where $x_{i}$ is a rotation phase ranging from 0 to $2 \pi$. Geometrically it means that for a fixed $S O(2 n)$ transformation, there is a basis in which this transformation looks like a set of rotations in independent two-planes. In the $S O(2 n+1)$ case the last column/row is zero. It corresponds to a fixed one-dimensional subspace. Nonnormalized Haar measure in these two cases reads as:

$$
\begin{aligned}
d \lambda_{S O(2 n)}= & \prod_{i<j}^{n} \sin \left(\frac{x_{i}-x_{j}}{2}\right)^{2} \sin \left(\frac{x_{i}+x_{j}}{2}\right)^{2} d x \\
d \lambda_{S O(2 n+1)}= & \prod_{i<j}^{n} \sin \left(\frac{x_{i}-x_{j}}{2}\right)^{2} \sin \left(\frac{x_{i}+x_{j}}{2}\right)^{2} \\
& \times \prod_{j=1}^{n} \sin \left(\frac{x_{j}}{2}\right)^{2} d x_{1} \ldots d x_{n} .
\end{aligned}
$$

Now we discuss the case where the gauge group is the product of three orthogonal groups $S O\left(N_{1}\right) \times S O\left(N_{2}\right) \times$ $S O\left(N_{3}\right)$, so that the gauge field decomposes as

$A=A^{1} \otimes \mathbb{1} \otimes \mathbb{1}+\mathbb{1} \otimes A^{2} \otimes \mathbb{1}+\mathbb{1} \otimes \mathbb{1} \otimes A^{3}$.

For even $N_{i}$ in Eq. (5.10) eigenvalues $\lambda_{a}$ are given by $x_{i}+$ $y_{j}+z_{k},-x_{i}+y_{j}+z_{k}, x_{i}-y_{j}+z_{k}$ and $x_{i}+y_{j}-z_{k}$, with $i=1, \ldots,\left\lfloor N_{1} / 2\right\rfloor, j=1, \ldots,\left\lfloor N_{2} / 2\right\rfloor, k=1, \ldots,\left\lfloor N_{3} / 2\right\rfloor$. 
TABLE II. Number of singlet states in the $O(N)^{6}$ GurauWitten model.

\begin{tabular}{cc}
\hline \hline$N$ & No. of singlet states \\
\hline 2 & 140 \\
3 & 63358 \\
4 & 114876653804156708 \\
\hline \hline
\end{tabular}

Variables $x_{i}, y_{j}, z_{k}$ are rotation phases for $S O\left(N_{1}\right), S O\left(N_{2}\right)$ and $S O\left(N_{3}\right)$ respectively. In the case when one of the $N_{i}$ is odd we have to add a zero eigenvalue to this list. With the use of the Eq. (5.10) we can write the explicit form of the character of the representation and decompose it in terms of characters of the irreducible representations. For example, for the $O(2)^{3}$ model the number of singlets is given by the integral

$$
\begin{aligned}
& \frac{16}{(2 \pi)^{3}} \int_{-\pi}^{\pi} d x \int_{-\pi}^{\pi} d y \int_{-\pi}^{\pi} d z \cos \left(\frac{x+y+z}{2}\right) \\
& \quad \times \cos \left(\frac{x+y-z}{2}\right) \cos \left(\frac{x-y+z}{2}\right) \cos \left(\frac{-x+y+z}{2}\right),
\end{aligned}
$$

whose evaluation gives 2 .

For the $O(N)^{3}$ model the number of singlets for various even $N$ is given in Table I. For odd $N$ it is not hard to see that the integral which gives the number of singlets vanishes; this is related to the fact that each group exhibits an individual anomaly, which we discuss in the next section. ${ }^{4}$ In the next Sec. VA we will show that the number of singlets grows as $\exp \left(N^{3} \log 2 / 2-3 N^{2} \log N / 2\right)$ for large even $N$.

Using similar methods, the number of singlets can be calculated in the $O(N)^{6} \mathrm{GW}$ model for low values of $N$, and the results are presented in Table II. The fact that there are 140 states for $N=2$ is in agreement with the direct construction of singlet states in [46].
TABLE III. Number of singlet states in the $O\left(N_{1}\right) \times O\left(N_{2}\right) \times$ $O(2)$ model.

\begin{tabular}{lc}
\hline \hline$\left(N_{1}, N_{2}\right)$ & No. of singlet states \\
\hline$(4,4)$ & 4 \\
$(6,4)$ & 4 \\
$(6,6)$ & 4 \\
$(8,4)$ & 6 \\
$(8,6)$ & 8 \\
$(8,8)$ & 18 \\
$(10,4)$ & 6 \\
$(10,6)$ & 8 \\
$(10,8)$ & 20 \\
$(10,10)$ & 24 \\
\hline \hline
\end{tabular}

We may similarly calculate the number of singlets for the $O\left(N_{1}\right) \times O\left(N_{2}\right) \times O\left(N_{3}\right)$ models. It is non-vanishing only if all $N_{i}$ are even. When $N_{2}=N_{3}=2$, while $N_{1}$ is even, there are 2 singlets. For the cases where $N_{3}=2$, while $N_{1}$ and $N_{2}$ are even, some answers are listed in Table III. We note that the growth of the number of singlets for the $O(N)^{2} \times O(2)$ model is much slower than for the $O(N)^{3}$ model. For low values of $N$ it is not hard to write down explicit expressions for all the singlet states in the oscillator basis; see Appendix B 3. For example, for the $O(4)^{2} \times$ $O(2)$ model we find that the 4 singlet energies are $\pm 16 g$ and $\pm 4 g$. The fact that the singlet spectrum is nondegenerate is related to the absence of discrete symmetries of the Hamiltonian in the minimal tensor model of [2]; this is in contrast to the $O(N)^{6} \mathrm{GW}$ model where the singlet eigenvalues have degeneracies [46].

\section{A. Number of singlets for large $N$}

In this section we will estimate the number of singlets in the $S O(N)^{3}$ model in the large $N$ limit, assuming $N$ to be odd $N=2 M$. For general $N$, the number of singlets is given by the following integral:

$$
\begin{aligned}
\text { singlet states }= & \frac{1}{V^{3}} \int_{-\pi}^{\pi}[d x][d y][d z] \prod_{i, j, k=1}^{M} 16 \cos \left(\frac{x_{i}+y_{j}+z_{k}}{2}\right) \cos \left(\frac{-x_{i}+y_{j}+z_{k}}{2}\right) \cos \left(\frac{x_{i}-y_{j}+z_{k}}{2}\right) \cos \left(\frac{x_{i}+y_{j}-z_{k}}{2}\right) \\
& \times \prod_{i<j}^{M} \sin ^{2}\left(\frac{x_{i}-x_{j}}{2}\right) \sin ^{2}\left(\frac{x_{i}+x_{j}}{2}\right) \sin ^{2}\left(\frac{y_{i}-y_{j}}{2}\right) \sin ^{2}\left(\frac{y_{i}+y_{j}}{2}\right) \sin ^{2}\left(\frac{z_{i}-z_{j}}{2}\right) \sin ^{2}\left(\frac{z_{i}+z_{j}}{2}\right)
\end{aligned}
$$

Where $V$ is the volume of $S O(N)$. When $N$ is large, cosine functions oscillate very rapidly, so the integral localizes near $x_{i}=y_{j}=z_{k}=0$. Near this point the integrand is positive, so we can exponentiate it:

\footnotetext{
${ }^{4}$ Direct diagonalization of the Hamiltonian for $N=3[41,42]$ reveals that there are no nondegenerate eigenvalues, consistent with this. There are 8 ground states with energy $-\frac{5}{4} \sqrt{41} g \approx-8.00391 g$; they transform in the spinorial $(2,2,2)$ representation. Substituting the value $C_{i}=3 / 4$ into the bound (3.21) for the energy gives $-11.53 \mathrm{~g}$, which is quite close to the actual value.
} 


$$
\begin{aligned}
\text { No. of singlet states }= & \int_{-\pi}^{\pi}[d x][d y][d z] \exp \left(4 \sum_{n=1}^{\infty} \sum_{i, j, k=1}^{M} \frac{(-1)^{n+1}}{n} t^{n} \cos \left(n x_{i}\right) \cos \left(n y_{j}\right) \cos \left(n z_{k}\right)\right) \\
& \times \prod_{i<j}^{M} \sin ^{2}\left(\frac{x_{i}-x_{j}}{2}\right) \sin ^{2}\left(\frac{x_{i}+x_{j}}{2}\right) \sin ^{2}\left(\frac{y_{i}-y_{j}}{2}\right) \sin ^{2}\left(\frac{y_{i}+y_{j}}{2}\right) \sin ^{2}\left(\frac{z_{i}-z_{j}}{2}\right) \sin ^{2}\left(\frac{z_{i}+z_{j}}{2}\right)
\end{aligned}
$$

Notice that we have introduced a "regulator" $t$ which we have to send to one: $t \rightarrow 1$. Similar integrals count operators in theories with trifundamental fields [36]. In such cases $t=e^{-1 / T}$, where $T$ is the temperature. So we are interested in the infinite temperature limit. This case has been studied in detail in [36]. Here we perform a similar analysis. As usual, we will encode the saddle-point configuration of the angles $x, y, z$ using the density function $\rho(x)$ (obviously it is the same function for the three $S O(N)$ groups). Moreover this function is symmetric $\rho(x)=\rho(-x)$. It would be convenient to work with the normalized density $\int_{-\pi}^{\pi} d x \rho(x)=1$. The effective action now reads as:

$$
\begin{aligned}
S[\rho]= & \frac{1}{2} N^{3} \int_{-\pi}^{\pi} d x d y d z \rho(x) \rho(y) \rho(z) \sum_{n=1}^{\infty} \frac{(-1)^{n+1} t^{n}}{n} \cos (n x) \cos (n y) \cos (n z) \\
& +\frac{1}{4} N^{2} \int_{-\pi}^{\pi} d x d x^{\prime} \rho(x) \rho\left(x^{\prime}\right) \log \sin \left(\frac{x-x^{\prime}}{2}\right)^{4}+\frac{1}{4} N^{2} \int_{-\pi}^{\pi} d y d y^{\prime} \rho(y) \rho\left(y^{\prime}\right) \log \sin \left(\frac{y-y^{\prime}}{2}\right)^{4} \\
& +\frac{1}{4} N^{2} \int_{-\pi}^{\pi} d z d z^{\prime} \rho(z) \rho\left(z^{\prime}\right) \log \sin \left(\frac{z-z^{\prime}}{2}\right)^{4}
\end{aligned}
$$

In the infinite temperature limit the saddle-point density is nonzero only on a small interval $\left[-x_{0}, x_{0}\right]$ where $x_{0} \sim \sqrt{\frac{2}{N}}$. The leading contribution is coming from the first term and it equals to $\frac{1}{2} N^{3} \log 2$. But this yields simply the dimensions of the Hilbert space, which is $2^{\frac{1}{2} N^{3}}$. The subleading term is coming from the second term in (5.18). Fortunately, we will not need the exact value of $x_{0}$ because of the logarithmic behavior:

$$
\begin{aligned}
\int_{-x_{0}}^{x_{0}} d x d x^{\prime} \rho(x) \rho\left(x^{\prime}\right) \log \sin \left(\frac{x-x^{\prime}}{2}\right)^{4} \sim 4 \int_{-x_{0}}^{x_{0}} d x d x^{\prime} \rho(x) \rho\left(x^{\prime}\right) \log \left(x-x^{\prime}\right) & \sim 4 \int_{-x_{0}}^{x_{0}} d x d x^{\prime} \rho(x) \rho\left(x^{\prime}\right) \log x_{0} \\
& =4 \log x_{0} \sim-2 \log N .
\end{aligned}
$$

Therefore the subleading term is $-\frac{3}{4} N^{2} \log N$. So, in total we have

$$
\text { No. of singlet states } \sim \exp \left(\frac{N^{3}}{2} \log 2-\frac{3 N^{2}}{2} \log N+O\left(N^{2}\right)\right) .
$$

\section{B. Anomalies}

Since we are studying fermions on a compact space $S^{1}$ there is a potential global anomaly associated with $\pi_{1}(G)$. And indeed it is well known that $\pi_{1}(S O(N))=\mathbb{Z}_{2}$. Corresponding "large" gauge transformation has a simple description: the gauge transformation matrix is the identity matrix, apart from one $2 \times 2$ block

$$
\left(\begin{array}{cc}
\cos (2 \pi t) & -\sin (2 \pi t) \\
\sin (2 \pi t) & \cos (2 \pi t)
\end{array}\right) .
$$

It is easy to see that after such transformation one chosen rotation phase $x_{i}$ will be shifted by $2 \pi: x_{i} \rightarrow x_{i}+2 \pi$. It does not matter which $x_{i}$ to pick up, since an even number of $2 \pi$-rotation blocks gives, in fact, a trivial element in $\pi_{1}(S O(N))$. It has been known for some time [53] that a theory of a single Majorana fermion in the fundamental representation of $S O(N)$ is suffering from this $\mathbb{Z}_{2}$ anomaly. It is instructive to see it using our machinery. The Pfaffian in this case reads as:

$$
\prod_{i=1}^{N / 2} \cos \left(x_{i} / 2\right) .
$$

Under the shift $x_{j} \rightarrow x_{j}+2 \pi$ it changes sign. Therefore the theory is not invariant under large gauge transformations. In our case of $O\left(N_{1}\right) \times O\left(N_{2}\right) \times O\left(N_{3}\right)$ group it means that at least two out of three $N_{i}$ should be even, otherwise we will have an odd number of anomalous multiplets. Since this anomaly is associated with only one group we will refer to it as "individual anomaly." It is easy to see that this anomaly is always $\mathbb{Z}_{2}$ (in other words, it squares to one), even if we add more gauge groups. 
If the gauge group is a product $S O\left(2 n_{1}\right) \times S O\left(2 n_{2}\right)$ there is a new anomaly mixing these two groups. For each group in the product, the large gauge transformation consists of identical $2 \times 2$ blocks:

$$
\left(\begin{array}{cc}
\cos (\pi t) & -\sin (\pi t) \\
\sin (\pi t) & \cos (\pi t)
\end{array}\right)
$$

Since there are two gauge groups, at $t=1$ overall -1 will cancel. Now all phases $x_{i}$ and $y_{j}$ are shifted by $\pi$ : $x_{i} \rightarrow x_{i}+\pi, y_{j} \rightarrow y_{j}+\pi$. The Pfaffian reads as:

$$
\prod_{i=1}^{n_{1}} \prod_{j=1}^{n_{2}} \cos \left(\frac{x_{i}+y_{j}}{2}\right) \cos \left(\frac{x_{i}-y_{j}}{2}\right) .
$$

Under the large gauge transformation the Pfaffian acquires $(-1)^{n_{1} n_{2}}$. This anomaly means that for $G=S O\left(2 n_{1}\right) \times$ $S O\left(2 n_{2}\right) \times S O\left(N_{3}\right), N_{3}$ can be odd only if the product $N_{1} N_{2}$ is even. We will call this anomaly "mixed anomaly." This anomaly is not always $\mathbb{Z}_{2}$ as we will see shortly.

We do not find any more anomalies: using the long exact sequence in homotopy groups one can show that the fundamental group of $S O\left(2 n_{1}\right) \times S O\left(2 n_{2}\right) / \mathbb{Z}_{2}{ }^{5}$ is equal to $\mathbb{Z}_{2} \times \mathbb{Z}_{2} \times \mathbb{Z}_{2}$ or $\mathbb{Z}_{4} \times \mathbb{Z}_{2}$ depending on $n_{1}$ and $n_{2}$. Using the above explicit descriptions of the individual anomalies and the mixed anomaly we see that:

(i) If $n_{1}$ and $n_{2}$ are both even, then the square of the mixed anomaly gives a trivial gauge transformation. Indeed, for each gauge group the number $N_{i}$ of $2 \pi$ rotation blocks (5.21) is even. Therefore, this is the case of $\mathbb{Z}_{2} \times \mathbb{Z}_{2} \times \mathbb{Z}_{2}$.

(ii) If only one of $n_{i}$, say $n_{1}$, is odd, then the mixed anomaly squares to the individual anomaly of $S O\left(2 n_{1}\right)$, since this group will have an odd number of $2 \pi$ rotation blocks. Therefore, the anomalies form $\mathbb{Z}_{4} \times \mathbb{Z}_{2}$.

(iii) Finally, when both $n_{1}$ and $n_{2}$ are odd, then the mixed anomaly squares to the sum of the individual anomalies. This is again $\mathbb{Z}_{4} \times \mathbb{Z}_{2}$.

\section{SOLUTION OF SOME FERMIONIC MATRIX MODELS}

When $N_{3}=1$ or $N_{3}=2$ the $O\left(N_{1}\right) \times O\left(N_{2}\right) \times O\left(N_{3}\right)$ symmetric tensor model (1.2) simplifies and becomes a fermionic $N_{1} \times N_{2}$ matrix model. In this section we discuss the solution of these models. For the $O\left(N_{1}\right) \times O\left(N_{2}\right)$ real matrix model the Hamiltonian may be expressed in terms of the quadratic Casimir operators, which shows that all the states within the same group representation have the same energy. This also applies to the $S U\left(N_{1}\right) \times S U\left(N_{2}\right) \times U(1)$ symmetric complex fermionic matrix model, which was

\footnotetext{
${ }^{5}$ One has to divide by $\mathbb{Z}_{2}$ because $g_{1} \times g_{2}$ acts on $\psi$ in the same way as $\left(-g_{1}\right) \times\left(-g_{2}\right)$.
}

considered in $[49,54]$ (see also [55]), and will be further discussed in Sec. VI B. However, the $O\left(N_{1}\right) \times O\left(N_{2}\right) \times$ $U(1)$ complex fermionic matrix model is more complicated in that there are energy splittings within the same representation of the symmetry group. Nevertheless, as we show in Sec. VIC this model is solvable.

\section{A. The $O\left(N_{1}\right) \times O\left(N_{2}\right)$ model}

Setting $N_{3}=1$ in the $O\left(N_{1}\right) \times O\left(N_{2}\right) \times O\left(N_{3}\right)$ symmetric tensor model (1.2) we find a real matrix model with $O\left(N_{1}\right) \times O\left(N_{2}\right)$ symmetry:

$H=\frac{g}{4} \psi^{a b} \psi^{a b^{\prime}} \psi^{a^{\prime} b} \psi^{a^{\prime} b^{\prime}}-\frac{g}{16} N_{1} N_{2}\left(N_{1}-N_{2}+1\right)$.

Using the $S O\left(N_{1}\right)$ and $S O\left(N_{2}\right)$ charges

$$
Q_{1}^{a a^{\prime}}=\frac{i}{2}\left[\psi^{a b}, \psi^{a^{\prime} b}\right], \quad Q_{2}^{b b^{\prime}}=\frac{i}{2}\left[\psi^{a b}, \psi^{a b^{\prime}}\right]
$$

the Hamiltonian may be expressed in terms of the quadratic Casimirs:

$$
\begin{aligned}
H & =-\frac{g}{2} C_{2}^{S O\left(N_{2}\right)}+\frac{g}{16} N_{1} N_{2}\left(N_{2}-1\right) \\
& =\frac{g}{2} C_{2}^{S O\left(N_{1}\right)}-\frac{g}{16} N_{1} N_{2}\left(N_{1}-1\right) .
\end{aligned}
$$

This shows that, under the interchange of $N_{1}$ and $N_{2}$, $H \rightarrow-H$; therefore, for $N_{1}=N_{2}$ the spectrum is symmetric around zero. The sum of this Casimir operators is fixed:

$$
\begin{aligned}
C_{2}^{S O\left(N_{1}\right)}+C_{2}^{S O\left(N_{2}\right)} & =\frac{1}{2} Q_{1}^{a a^{\prime}} Q_{1}^{a a^{\prime}}+\frac{1}{2} Q_{2}^{b b^{\prime}} Q_{2}^{b b^{\prime}} \\
& =\frac{1}{8} N_{1} N_{2}\left(N_{1}+N_{2}-2\right) .
\end{aligned}
$$

This shows that there are no states which are singlets under both $S O\left(N_{1}\right)$ and $S O\left(N_{2}\right)$. The irreducible representations $\left(r_{1}, r_{2}\right)$ which appear in the spectrum must satisfy the condition (6.4). In Appendix B 1 we list these representations for a few low values of $N_{1}$ and $N_{2}$. The complete lists of the energies and degeneracies are shown in Table IV.

For $O(N) \times O(N)$ with even $N$, we find that the ground state is a singlet under $O(N)_{1}$ and transforms in the $S O(N)_{2}$

TABLE IV. Spectra of the $O\left(N_{1}\right) \times O\left(N_{2}\right)$ models.

\begin{tabular}{lrrrrrrr}
\hline \hline$\left(N_{1}, N_{2}\right)$ & $(2,2)$ & $(2,3)$ & $(2,4)$ & $(3,3)$ & $(3,4)$ & $(4,4)$ & $(5,5)$ \\
\hline$\frac{4}{g} E_{\text {degeneracy }}$ & $-1_{2}$ & $-1_{6}$ & $-2_{6}$ & $-3_{8}$ & $-6_{8}$ & $-12_{10}$ & $-20_{224}$ \\
& $1_{2}$ & $3_{2}$ & $0_{8}$ & $3_{8}$ & $-2_{36}$ & $-6_{64}$ & $-10_{1024}$ \\
& & & $6_{2}$ & & $6_{20}$ & $-4_{54}$ & $-4_{800}$ \\
& & & & & & $4_{54}$ & $4_{800}$ \\
& & & & & & $6_{64}$ & $10_{1024}$ \\
& & & & & & & $22_{224}$ \\
\hline \hline
\end{tabular}


representation whose Young diagram is a $\frac{N}{2} \times \frac{N}{2}$ square. The ground state has energy $E_{0}=-g N^{2}(N-1) / 16$, while the first excited state is in the fundamental of $O(N)_{1}$ which has quadratic Casimir $N-1$. Therefore, the energy gap

$$
E_{1}-E_{0}=\frac{g}{2}(N-1) .
$$

In the 't Hooft large $N$ limit, $g \sim 1 / N$ and the gap stays finite. Therefore, unlike the SYK and tensor models, the matrix model cannot exhibit quasiconformal behavior.

\section{B. The $S U\left(N_{1}\right) \times S U\left(N_{2}\right) \times U(1)$ model}

In [2] a class of complex tensor quantum mechanical models with $S U\left(N_{1}\right) \times S U\left(N_{2}\right) \times O\left(N_{3}\right) \times U(1)$ symmetry was introduced. We will use the Hamiltonian

$$
\begin{aligned}
H= & g \bar{\psi}_{a b c} \bar{\psi}_{a^{\prime} b^{\prime} c} \psi_{a b^{\prime} c^{\prime}} \psi_{a^{\prime} b c^{\prime}}+g\left(N_{1}-N_{2}\right) Q \\
& +\frac{g}{4} N_{1} N_{2} N_{3}\left(N_{1}-N_{2}\right),
\end{aligned}
$$

where $\psi_{a b c}$ with $a=1, \ldots, N_{1}, b=1, \ldots, N_{2}$ and $c=$ $1, \ldots, N_{3}$ are complex fermions with anti-commutation relations $\left\{\bar{\psi}_{a b c}, \psi_{a^{\prime} b^{\prime} c^{\prime}}\right\}=\delta_{a a^{\prime}} \delta_{b b^{\prime}} \delta_{c c^{\prime}}$. The second and third terms were added to the Hamiltonian to make it traceless and invariant under the charge conjugation symmetry, which interchanges $\psi_{a b c}$ and $\bar{\psi}_{a b c}$. This means it is invariant under $Q \rightarrow-Q$, where $Q$ is the $U(1)$ charge:

$$
Q=\bar{\psi}_{a b c} \psi_{a b c}-\frac{1}{2} N_{1} N_{2} N_{3} .
$$

If we set $N_{3}=1$ we obtain a complex matrix model with $S U\left(N_{1}\right) \times S U\left(N_{2}\right) \times U(1)$ symmetry $^{6}$

$$
\begin{aligned}
H= & g \bar{\psi}_{a b} \bar{\psi}_{a^{\prime} b^{\prime}} \psi_{a b^{\prime}} \psi_{a^{\prime} b}+g\left(N_{1}-N_{2}\right) Q \\
& +\frac{g}{4} N_{1} N_{2}\left(N_{1}-N_{2}\right),
\end{aligned}
$$

which is the subject of this section. Note that the index contraction in the first term is different from those in (6.20); the $S U\left(N_{1}\right) \times S U\left(N_{2}\right) \times U(1)$ symmetry fixes it uniquely. This matrix model has some features in common with the $O\left(N_{1}\right) \times O\left(N_{2}\right)$ from the previous section. In both of them the energy is completely fixed by the quadratic Casimir operators of the symmetry group factors. Also, neither model contains states invariant under the entire symmetry group.

The $S U\left(N_{i}\right)$ charges with $i=1,2$ are

$$
\begin{aligned}
Q_{1}^{\alpha} & =\bar{\psi}_{a b}\left(T_{1}^{\alpha}\right)_{a a^{\prime}} \psi_{a^{\prime} b}, \\
Q_{2}^{\alpha} & =\bar{\psi}_{a b}\left(T_{2}^{\alpha}\right)_{b b^{\prime}} \psi_{a b^{\prime}}, \\
\alpha & =1,2, \ldots, N_{i}^{2}-1,
\end{aligned}
$$

where we used the Hermitian $S U\left(N_{i}\right)$ generators $T_{i}^{\alpha}, i=1$, $2, \alpha=1, \ldots, N_{i}^{2}-1$, normalized in the standard fashion:

\footnotetext{
${ }^{6}$ This Hamiltonian is related to that in Sec. IV of [49] by changing the coefficients of the second and third terms.
}

$$
\operatorname{Tr}\left(T_{1}^{\alpha} T_{1}^{\beta}\right)=\operatorname{Tr}\left(T_{2}^{\alpha} T_{2}^{\beta}\right)=\frac{1}{2} \delta^{\alpha \beta}
$$

Using the completeness relation (no sum over $i$ ):

$$
\left(T_{i}^{\alpha}\right)_{a a^{\prime}}\left(T_{i}^{\alpha}\right)_{b b^{\prime}}=\frac{1}{2}\left(\delta_{a b^{\prime}} \delta_{a^{\prime} b}-\frac{1}{N_{i}} \delta_{a a^{\prime}} \delta_{b b^{\prime}}\right) .
$$

we find that the quadratic Casimirs of $S U\left(N_{2}\right)$ and $S U\left(N_{2}\right)$ :

$$
\begin{aligned}
C_{2}^{S U\left(N_{1}\right)}=Q_{1}^{\alpha} Q_{1}^{\alpha}= & \frac{1}{2} \bar{\psi}_{a b} \bar{\psi}_{a^{\prime} b^{\prime}} \psi_{a b^{\prime}} \psi_{a^{\prime} b}+\frac{1}{2}\left(N_{1}-N_{2}\right) Q \\
& -\frac{1}{2 N_{1}} Q^{2}+\frac{1}{8} N_{1} N_{2}\left(2 N_{1}-N_{2}\right), \\
C_{2}^{S U\left(N_{2}\right)}=Q_{2}^{\alpha} Q_{2}^{\alpha}= & -\frac{1}{2} \bar{\psi}_{a b} \bar{\psi}_{a^{\prime} b^{\prime}} \psi_{a b^{\prime}} \psi_{a^{\prime} b}+\frac{1}{2}\left(N_{2}-N_{1}\right) Q \\
& -\frac{1}{2 N_{2}} Q^{2}+\frac{1}{8} N_{1} N_{2}\left(2 N_{2}-N_{1}\right) .
\end{aligned}
$$

Adding them, we obtain the constraint

$$
C_{2}^{S U\left(N_{1}\right)}+C_{2}^{S U\left(N_{2}\right)}=\frac{N_{1}+N_{2}}{2 N_{1} N_{2}}\left(\frac{\left(N_{1} N_{2}\right)^{2}}{4}-Q^{2}\right) .
$$

To have the singlets of $S U\left(N_{1}\right)$ and $S U\left(N_{2}\right)$, we need the RHS to vanish. This means that there are only two $S U\left(N_{1}\right) \times S U\left(N_{2}\right)$ singlet states: the ones with $Q=$ $\pm \frac{N_{1} N_{2}}{2}$. These are the oscillator vacuum $|0\rangle$, which is annihilated by all $\psi_{a b}$, and the state $\left|0^{\prime}\right\rangle=\prod_{a, b} \bar{\psi}_{a b}|0\rangle$, which is annihilated by all $\bar{\psi}_{a b}$.

The absence of singlets for other values of $Q$ may be seen explicitly as follows. The states with charge $-\frac{N_{1} N_{2}}{2}+m$ have the form

$$
\bar{\psi}_{a_{1} b_{1}} \bar{\psi}_{a_{2} b_{2}} \ldots \bar{\psi}_{a_{m} b_{m}}|0\rangle,
$$

but there is no way to contract the indices of $S U\left(N_{1}\right)$ and of $S U\left(N_{2}\right)$; in contrast to the $O(N)$ case, the tensor $\delta_{a_{1} a_{2}}$ is not available. If $N_{1}=N_{2}=N$ there seems to be a state at level $N$ obtained by contracting (6.14) with $\epsilon_{a_{1} \ldots a_{N}} \epsilon_{b_{1} \ldots b_{N}}$, but this state vanishes due to the Fermi statistics.

Using (6.12) we can express the Hamiltonian (6.8) in terms of the Casimirs:

$$
H=g\left(2 C_{2}^{S U\left(N_{1}\right)}+\frac{1}{N_{1}} Q^{2}-\frac{1}{4} N_{1}^{2} N_{2}\right) .
$$

Therefore, all the states in the same representation of $S U\left(N_{1}\right) \times S U\left(N_{2}\right) \times U(1)$ are degenerate, which makes this matrix model very simple. In Table $\mathrm{V}$ we list the spectra of the Hamiltonian (6.8) for a few different values of $N_{1}$ and $N_{2}$. 
TABLE V. Spectra of the $S U\left(N_{1}\right) \times S U\left(N_{2}\right) \times U(1)$ symmetric matrix models.

\begin{tabular}{lcccc}
\hline \hline$\left(N_{1}, N_{2}\right)$ & $(1,2)$ & $(1,3)$ & $(2,2)$ & $(2,3)$ \\
\hline$\frac{2}{g} E_{\text {degeneracy }}$ & $-1_{2}$ & $-1_{6}$ & $-4_{3}$ & $-5_{12}$ \\
& $1_{2}$ & $3_{2}$ & $0_{10}$ & $-3_{16}$ \\
& & & $4_{3}$ & $1_{12}$ \\
& & & $3_{20}$ \\
& & & $9_{4}$ \\
\hline \hline
\end{tabular}

\section{The $O\left(N_{1}\right) \times O\left(N_{2}\right) \times U(1)$ model}

Setting $N_{3}=2$ in the $O\left(N_{1}\right) \times O\left(N_{2}\right) \times O\left(N_{3}\right)$ symmetric tensor model (1.2) we find a complex matrix model with $O\left(N_{1}\right) \times O\left(N_{2}\right) \times U(1)$ symmetry. This model has some features in common with the $S U\left(N_{1}\right) \times S U\left(N_{2}\right) \times$ $U(1)$ model discussed in the previous section; they possess the same $2^{N_{1} N_{2}}$ dimensional Hilbert space. However, in the present model the symmetry is broken to $O\left(N_{1}\right) \times O\left(N_{2}\right) \times$ $U(1)$ by the Hamiltonian. Although the model is still exactly solvable, it is quite interesting in that the energy is not completely fixed by the quadratic Casimir operators of $O\left(N_{1}\right) \times O\left(N_{2}\right) \times U(1)$. Also, as we have seen in Sec. V, for even $N_{1}$ and $N_{2}$ the model contains singlet states.

To construct the Hilbert space, we define the operators [44]

$$
\begin{aligned}
\bar{\psi}_{a b} & =\frac{1}{\sqrt{2}}\left(\psi^{a b 1}+i \psi^{a b 2}\right), \quad \psi_{a b}=\frac{1}{\sqrt{2}}\left(\psi^{a b 1}-i \psi^{a b 2}\right), \\
\left\{\bar{\psi}_{a b}, \bar{\psi}_{a^{\prime} b^{\prime}}\right\} & =\left\{\psi_{a b}, \psi_{a^{\prime} b^{\prime}}\right\}=0, \quad\left\{\bar{\psi}_{a b}, \psi_{a^{\prime} b^{\prime}}\right\}=\delta_{a a^{\prime}} \delta_{b b^{\prime}},
\end{aligned}
$$

where $a=1,2, \ldots N_{1}$ and $b=1,2 \ldots N_{2}$. In this basis, the $O(2)$ charge is

$$
\begin{aligned}
Q & =\frac{1}{2}\left[\bar{\psi}_{a b}, \psi_{a b}\right]=\bar{\psi}_{a b} \psi_{a b}-\frac{1}{2} N_{1} N_{2}, \\
{\left[Q, \bar{\psi}_{a b}\right] } & =\bar{\psi}_{a b}, \quad\left[Q, \psi_{a b}\right]=-\psi_{a b},
\end{aligned}
$$

while the $S O\left(N_{1}\right)$ and $S O\left(N_{2}\right)$ charges are

$$
\begin{aligned}
& Q_{1}^{a a^{\prime}}=i\left(\bar{\psi}_{a b} \psi_{a^{\prime} b}-\bar{\psi}_{a^{\prime} b} \psi_{a b}\right), \\
& Q_{2}^{b b^{\prime}}=i\left(\bar{\psi}_{a b} \psi_{a b^{\prime}}-\bar{\psi}_{a b^{\prime}} \psi_{a b}\right) .
\end{aligned}
$$

Squaring these charges, we find the following expressions for quadratic Casimirs:

$$
\begin{aligned}
C_{2}^{O\left(N_{1}\right)}=\frac{1}{2} Q_{1}^{a a^{\prime}} Q_{1}^{a a^{\prime}}= & \bar{\psi}_{a b} \bar{\psi}_{a b^{\prime}} \psi_{a^{\prime} b} \psi_{a^{\prime} b^{\prime}}+\bar{\psi}_{a b} \bar{\psi}_{a^{\prime} b^{\prime}} \psi_{a b^{\prime}} \psi_{a^{\prime} b} \\
& +\left(N_{1}-1\right)\left(Q+\frac{1}{2} N_{1} N_{2}\right) \\
C_{2}^{O\left(N_{2}\right)}=\frac{1}{2} Q_{2}^{b b^{\prime}} Q_{2}^{b b^{\prime}}= & \bar{\psi}_{a b} \bar{\psi}_{a^{\prime} b} \psi_{a b^{\prime}} \psi_{a^{\prime} b^{\prime}}-\bar{\psi}_{a b} \bar{\psi}_{a^{\prime} b^{\prime}} \psi_{a b^{\prime}} \psi_{a^{\prime} b} \\
& +\left(N_{2}-1\right)\left(Q+\frac{1}{2} N_{1} N_{2}\right)
\end{aligned}
$$

Setting $k=1$ in (3.12), we find that the traceless form of the Hamiltonian is

$$
\begin{aligned}
H= & \frac{g}{2}\left(\bar{\psi}_{a b} \bar{\psi}_{a b^{\prime}} \psi_{a^{\prime} b} \psi_{a^{\prime} b^{\prime}}-\bar{\psi}_{a b} \bar{\psi}_{a^{\prime} b} \psi_{a b^{\prime}} \psi_{a^{\prime} b^{\prime}}\right) \\
& +\frac{g}{8} N_{1} N_{2}\left(N_{2}-N_{1}\right) .
\end{aligned}
$$

This Hamiltonian exhibits the charge conjugation symmetry which acts as $\bar{\psi}_{a b} \leftrightarrow \psi_{a b}$. This means that states with opposite eigenvalues of $Q$ have the same energy.

There is a "Clifford vacuum" state, which satisfies

$$
\begin{aligned}
\psi_{a b}|0\rangle & =0, \quad Q|0\rangle=-\frac{N_{1} N_{2}}{2}|0\rangle, \\
H|0\rangle & =\frac{g}{8} N_{1} N_{2}\left(N_{2}-N_{1}\right)|0\rangle .
\end{aligned}
$$

There is also the conjugate vacuum $\left|0^{\prime}\right\rangle=\prod_{a b} \bar{\psi}_{a b}|0\rangle$ which satisfies

$$
\begin{aligned}
\bar{\psi}_{a b}\left|0^{\prime}\right\rangle & =0, \quad Q\left|0^{\prime}\right\rangle=\frac{N_{1} N_{2}}{2}\left|0^{\prime}\right\rangle, \\
H\left|0^{\prime}\right\rangle & =\frac{g}{8} N_{1} N_{2}\left(N_{2}-N_{1}\right)\left|0^{\prime}\right\rangle .
\end{aligned}
$$

Both of these states are invariant not only under $O\left(N_{1}\right) \times O\left(N_{2}\right)$, but under the enhanced symmetry $O\left(N_{1} N_{2}\right)$. It is interesting to note that the states $|0\rangle$ and $\left|0^{\prime}\right\rangle$ saturate the energy bound (3.21). Indeed, substituting $N_{3}=2, C_{2}^{O\left(N_{3}\right)}=Q^{2}=\left(N_{1} N_{2}\right)^{2} / 4, C_{2}^{O\left(N_{1}\right)}=C_{2}^{O\left(N_{2}\right)}=0$ into that equation we find $|E| \leq \frac{g}{8} N_{1} N_{2}\left|N_{2}-N_{1}\right|$. In fact, the bound obtained from (3.8) completely fixes the energy to be $\frac{g}{8} N_{1} N_{2}\left(N_{2}-N_{1}\right)$ because the states are $O\left(N_{1} N_{2}\right)$ invariant and $C_{2}^{O\left(N_{1} N_{2}\right)}=0$.

The states with vanishing $O(2)$ charge $Q$ are obtained by acting on $|0\rangle$ with $\frac{N_{1} N_{2}}{2}$ creation operators $\bar{\psi}_{a b}$. Then, to insure that the state is also a singlet under $S O\left(N_{1}\right) \times S O\left(N_{2}\right)$, we have to contract the indices using the invariant tensors $\epsilon_{a_{1}, \ldots a_{N_{1}}}, \delta_{a_{1} a_{2}}$ and $\epsilon_{b_{1}, \ldots b_{N_{2}}}, \delta_{b_{1} b_{2}}$. Some states invariant under $S O\left(N_{1}\right) \times S O\left(N_{2}\right) \times O(2)$ are listed in Appendix B 3.

For low values of $N_{1}$ and $N_{2}$ it is possible to construct the complete spectrum via direct numerical diagonalization. If $N_{1}=N_{2}$ or if one or both $N_{i}$ are equal to 2, the spectrum is symmetric under $E \rightarrow-E$ due to the fact that the interchange of two $O(N)$ groups send $H \rightarrow-H$. For all other values of $N_{i}$ the spectrum is not symmetric under $E \rightarrow-E$. The results for some low values of $N_{1}, N_{2}$ are shown in Table VI. For the $O(4)^{2} \times O(2)$ model the spectrum is plotted in Fig. 1.

A remarkable feature of the spectra is that all the eigenvalues of $4 \mathrm{H} / \mathrm{g}$ are integers. This suggests that this fermionic matrix model is exactly solvable for any $N_{1}$ and $N_{2}$. This is indeed the case, as we now show. The Hilbert 
TABLE VI. Spectra of the $O\left(N_{1}\right) \times O\left(N_{2}\right) \times O(2)$ models, which were obtained by a direct matrix diagonalization of the Hamiltonian (3.12) whose spectrum is traceless. If both $N_{1}$ and $N_{2}$ are even, the ground state is nondegenerate and is therefore a singlet.

\begin{tabular}{|c|c|c|c|c|c|c|}
\hline$\left(N_{1}, N_{2}\right)$ & $(2,2)$ & $(2,3)$ & $(3,3)$ & $(2,4)$ & $(3,4)$ & $(4,4)$ \\
\hline$\frac{4}{g} E_{\text {degeneracy }}$ & $\begin{array}{c}-8_{1} \\
0_{14} \\
8_{1}\end{array}$ & $\begin{array}{c}-13_{2} \\
-7_{6} \\
-3_{2} \\
-1_{22} \\
1_{22} \\
3_{2} \\
7_{6} \\
13_{2}\end{array}$ & $\begin{array}{c}-20_{6} \\
-16_{18} \\
-12_{16} \\
-8_{60} \\
-4_{42} \\
0_{228} \\
4_{42} \\
8_{60} \\
12_{16} \\
16_{18} \\
20_{6}\end{array}$ & $\begin{array}{c}-24_{1} \\
-16_{2} \\
-12_{16} \\
-8_{23} \\
-4_{16} \\
0_{140} \\
4_{16} \\
8_{23} \\
12_{16} \\
16_{2} \\
24_{1}\end{array}$ & $\begin{array}{c}-34_{6} \\
-28_{24} \\
-24_{8} \\
-22_{76} \\
-20_{40} \\
-18_{14} \\
-16_{152} \\
-14_{168} \\
-12_{40} \\
-10_{170} \\
-8_{240} \\
-6_{194} \\
-4_{384} \\
-2_{270} \\
0_{248} \\
2_{640} \\
4_{384} \\
6_{76} \\
8_{312} \\
10_{216} \\
14_{32} \\
16_{128} \\
18_{168} \\
20_{64} \\
26_{10} \\
28_{24} \\
30_{6} \\
38_{2}\end{array}$ & $\begin{array}{l}-64_{1} \\
-48_{55} \\
-40_{106} \\
-36_{256} \\
-32_{810} \\
-28_{256} \\
-24_{3250} \\
-20_{1024} \\
-16_{4985} \\
-12_{3072} \\
-8_{8932} \\
-4_{3584} \\
0_{12874} \\
4_{3584} \\
8_{8932} \\
12_{3072} \\
16_{4985} \\
20_{1024} \\
24_{3250} \\
28_{256} \\
32_{810} \\
36_{256} \\
40_{106} \\
48_{55} \\
64_{1}\end{array}$ \\
\hline
\end{tabular}

space can be constructed by repeatedly acting with $\bar{\psi}_{a b}$ on the vacuum state $|0\rangle$. One can group the $a, b$ indices into a multi-index $A$, ranging from 1 to $N_{1} N_{2}$. The commutation relations are invariant under the action of $S U\left(N_{1} N_{2}\right)$ on the Hilbert space, which preserves the commutation relations. Let us notice that the first term of Hamiltonian (3.12) is invariant under $S U\left(N_{1}\right) \times O\left(N_{2}\right) \times U(1)$, while the second under $O\left(N_{1}\right) \times S U\left(N_{2}\right) \times U(1)$ groups. Therefore, the full Hamiltonian is invariant only under the action of $O\left(N_{1}\right) \times$ $O\left(N_{2}\right) \times$ group. The complete Hilbert space is transformed under the $S U\left(N_{1} N_{2}\right)$ group that can be split into $S U\left(N_{1}\right) \times$ $S U\left(N_{2}\right)$ representations. In each representation $R$ under $S U\left(N_{2}\right)$, operators $Q_{2}^{\alpha}$ act by matrices $\left(T_{2}^{\alpha}\right)_{R}$ in the corresponding representation $R$. In turn, these representations can be split into $S O\left(N_{1}\right) \times S O\left(N_{2}\right)$ irreducible representations. Since the Hamiltonian has only $S O\left(N_{1}\right) \times$ $\mathrm{SO}\left(N_{2}\right)$ symmetry, all the states in such a representation are degenerate (of course, not all the states in a given $S U\left(N_{1}\right) \times S U\left(N_{2}\right)$ representation are in general degenerate).

Now we take the difference between Eq. (6.19), and also use the difference of equations (6.12), to find the following nice expression for the Hamiltonian:

$$
\begin{aligned}
H= & -\frac{g}{2}\left(2 C_{2}^{S U\left(N_{1}\right)}-2 C_{2}^{S U\left(N_{2}\right)}-C_{2}^{S O\left(N_{1}\right)}+C_{2}^{S O\left(N_{2}\right)}\right. \\
& \left.+\frac{N_{2}-N_{1}}{N_{1} N_{2}} Q^{2}+\left(N_{2}-N_{1}\right) Q\right) \\
= & -\frac{g}{2}\left(4 C_{2}^{S U\left(N_{1}\right)}-C_{2}^{S O\left(N_{1}\right)}+C_{2}^{S O\left(N_{2}\right)}+\frac{2}{N_{1}} Q^{2}\right. \\
& \left.+\left(N_{2}-N_{1}\right) Q-\frac{1}{4} N_{1} N_{2}\left(N_{1}+N_{2}\right)\right)
\end{aligned}
$$

where we used (6.13) to obtain the second line from the first. Due to the $C_{2}^{S O\left(N_{i}\right)}$ terms, the spectrum is not symmetric under $S U\left(N_{1}\right) \times S U\left(N_{2}\right)$.

Using (6.23) we can show that the lowest singlet saturates the energy bound (3.23), i.e. it is a ground state. For a singlet, $Q$ and the quadratic Casimir operators of $S O\left(N_{1}\right)$ and $S O\left(N_{2}\right)$ vanish. To minimize the energy we should take a state which has the greatest possible value of $C_{2}^{S U\left(N_{1}\right)}$ allowed by (6.13). Thus, it has $C_{2}^{S U\left(N_{1}\right)}=$ $\frac{\left(N_{1}+N_{2}\right) N_{1} N_{2}}{8}$ and $C_{2}^{S U\left(N_{2}\right)}=0$, i.e. it is invariant under $S O\left(N_{1}\right) \times S U\left(N_{2}\right) \times O(2)$. Substituting this into (6.23)

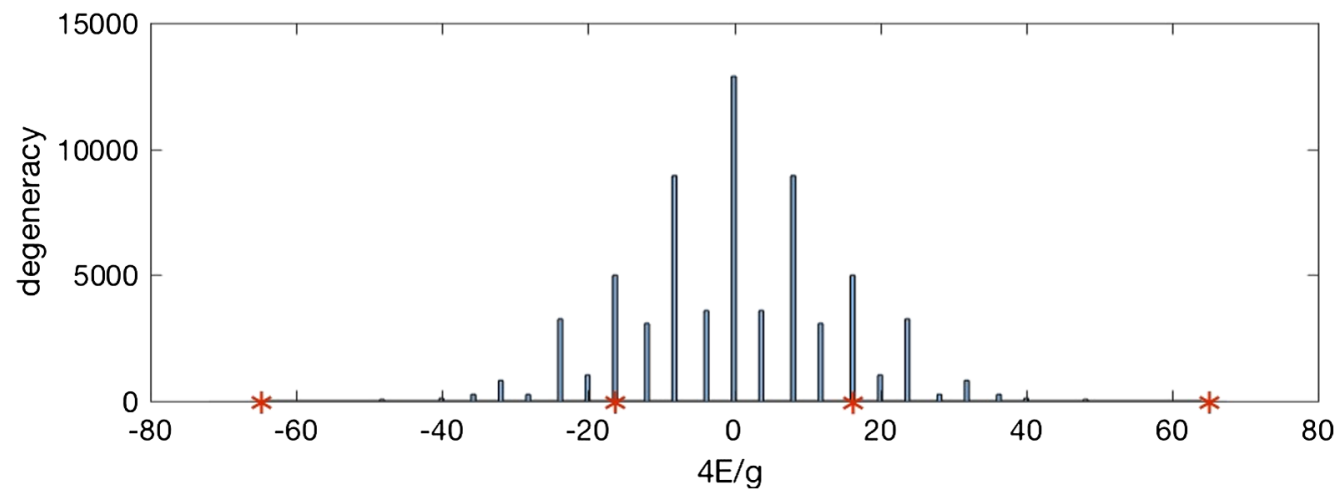

FIG. 1. Spectrum of the $O(4)^{2} \times O(2)$ model. There are four singlet states, and the stars mark their energies. 
we see that this state has $E=-\frac{g}{8}\left(N_{1}+N_{2}\right) N_{1} N_{2}$, i.e. it saturates the bound (3.23). This value of Casimir corresponds to the rectangular Young diagram $\left\lfloor N_{1} / 2\right\rfloor \times N_{2}$ for $S U\left(N_{1}\right)$. Similarly, the singlet state with the highest possible energy, $E=\frac{g}{8}\left(N_{1}+N_{2}\right) N_{1} N_{2}$, has $C_{2}^{S U\left(N_{2}\right)}=$ $\frac{\left(N_{1}+N_{2}\right) N_{1} N_{2}}{8}$ and $C_{2}^{S U\left(N_{1}\right)}=0$, i.e. it is invariant under $S U\left(N_{1}\right) \times S O\left(N_{2}\right) \times O(2)$.

To calculate the energies of all states, we need to first decompose the Hilbert space into $S U\left(N_{1}\right)_{L} \times S U\left(N_{2}\right)_{R}$ representations and then, in turn, decompose these representations into $S O\left(N_{1}\right)_{L} \times S O\left(N_{2}\right)_{R}$ representations. To find which $S U\left(N_{1}\right)_{L} \times S U\left(N_{2}\right)_{R}$ representations $(L, R)$ we have in the Hilbert space, we need to compute the following integral over $S U\left(N_{1}\right)_{L} \times S U\left(N_{2}\right)_{R}$ :

multiplicity $(L, R)$

$$
\begin{aligned}
= & \int d U_{1} d U_{2} \exp \left(\sum_{n=1}^{\infty} \frac{(-1)^{n+1}}{n} \operatorname{Tr} U_{1}^{n} \operatorname{Tr} U_{2}^{n}\right) \\
& \times \operatorname{Tr}_{L} U_{1} \operatorname{Tr}_{R} U_{2}
\end{aligned}
$$

We can always put $U_{1}$ and $U_{2}$ in a diagonal form: $U_{1}=\operatorname{diag}\left(w_{1}, \ldots, w_{N_{1}}\right), U_{2}=\operatorname{diag}\left(q_{1}, \ldots, q_{N_{2}}\right) . w_{i}$ and $q_{i}$ are corresponding $S U$ holonomies, i.e. $\left|w_{i}\right|=\left|q_{i}\right|=1$ and $w_{1} \ldots w_{N_{1}}=q_{1} \ldots q_{N_{2}}=1$.

Actually, it is not necessary to compute the above integral for various representations. It is very well-known that characters of $S U\left(N_{1}\right)$ representations are Schur polynomials $\operatorname{Tr}_{L} U_{1}=\chi_{L}(w)$ which form a basis in the space of symmetric functions of $N_{1}$ variables. This space also contains the so-called power series polynomials $\operatorname{Tr} U_{1}^{n}=$ $p_{n}(w)=w_{1}^{n}+\cdots+w_{N_{1}}^{n}$. A conversion from power series $p_{n}$ to $\chi_{L}$ can be easily done on a computer. For example,

$$
\begin{gathered}
p_{1}=\chi_{\square}, \quad p_{1}^{2}=\chi_{\square}+\chi_{\square}, \\
p_{2}=\chi_{\square}-\chi_{\square}, \quad p_{1} p_{2}=\chi_{\square \square}-\chi_{\square} .
\end{gathered}
$$

This suggests the following simple procedure yielding the list of all representations directly. One expands the exponent

$$
\begin{aligned}
\exp & \left(\sum_{n=1}^{\infty} \frac{(-1)^{n+1}}{n} x^{n} \operatorname{Tr} U_{1}^{n} \operatorname{Tr} U_{2}^{n}\right) \\
& =\exp \left(\sum_{n=1}^{\infty} \frac{(-1)^{n+1}}{n} x^{n} p_{n}(w) p_{n}(q)\right)
\end{aligned}
$$

in power series in $x$. Then at each level $x^{k}$ we have a polynomial in $p_{l}(w)$ and $p_{m}(q)$. It can be reexpressed in terms of Schur polynomials. This gives the list of representations under $S U_{L}\left(N_{1}\right) \times S U_{R}\left(N_{2}\right)$ at level $k$, i.e. for states where there are $k$ raising operators $\bar{\psi}$ acting on the vacuum.
After finding the representations under $S U\left(N_{1}\right)_{L} \times$ $S U\left(N_{2}\right)_{R}$, we need to decompose then in terms of $S O\left(N_{1}\right)_{L} \times S O\left(N_{2}\right)_{R}$ representations. Recall that both $S U$ and $S O$ representations are classified by Young diagrams. The only difference is that for $S O$ representations one has to subtract all the traces in each row, where indices are symmetric. It means that if we want to extract $S O$ representations from a given $S U$ representation $\lambda$, we need to consecutively remove all possible pairs of boxes in each row. The resulting sequence of Young diagrams give $S O$ representations.

Let us exhibit this method to find the spectrum of the $O(2)^{3}$ model. We have the following representations under $S U(2)_{L} \times S U(2)_{R}{ }^{7}$ :

$$
2([1],[1])+2([2],[2])+([1],[3])+([3],[1]) .
$$

The [2] of $S U(2)$ gives the spin $1 S O(2)$ representation, whereas the [3] decomposes as [3] $=2+0$. So we have the following $S O(2) \times S O(2)$ representations:

$$
2(0,0)+2(1,1)+2(0,0)+(0,2)+(2,0) .
$$

The two states $(0,0)$ coming from ([1], [3]) and ([3], [1]) have energies $\pm 2 g$, while all the other states have energy zero. If we label the states by their $O(2)^{3}$ charges $\left(Q_{1}, Q_{2}, Q_{3}\right)$, we find, in agreement with [43], that the states with $E= \pm 2 g$ are $(0,0,0)$, while the 14 zero-energy states are

$$
\begin{aligned}
& (1,1,1),(0,0,2),(0,2,0),(2,0,0),(1,1,-1),(1,-1,1), \\
& (-1,1,1),(-1,-1,-1),(0,0,-2),(0,-2,0), \\
& (-2,0,0),(-1,-1,1),(-1,1,-1),(1,-1,-1) .
\end{aligned}
$$

These states may be decomposed into irreducible representations of the alternating group $A_{3}$. For example, the state with charges $(1,1,1)$ is invariant under $A_{3}$; the 3 states with charges $(0,0,2),(0,2,0),(2,0,0)$ can be combined into an invariant combination and a dimension 2 representation; etc.

As a further check, in Appendix B 2 we calculate the spectrum of the $O(3) \times O(2) \times O(2)$ model using this method. The results for the energies and their degeneracies agree with the direct diagonalization of the Hamiltonian, whose results are assembled in Table VI. We also note that, due to the charge conjugation symmetry, the energies and representations at oscillator level $n$ are the same as at level $N_{1} N_{2}-n$.

\footnotetext{
${ }^{7}$ Here we are using the notation multiplicity $\left([\operatorname{dim}]_{L},[\operatorname{dim}]_{R}\right)$ for the $S U(2)_{L} \times S U(2)_{R}$ representations and multiplicity $\left(\operatorname{spin}_{L}, \operatorname{spin}_{R}\right)$ for $S O(2)_{L} \times S O(2)_{R}$ representations. For nonzero spin $J$, the $S O(2)$ representation is two-dimensional and includes the states with $S O(2)$ charge $Q= \pm J$.
} 


\section{ACKNOWLEDGMENTS}

We are grateful to Ksenia Bulycheva for collaboration at the early stages of this project. We also thank Dio Anninos, Andrei Bernevig, Sylvain Carrozza, Chethan Krishnan, Juan Maldacena, Daniel Roberts, Douglas Stanford and Edward Witten for useful discussions. The work of IRK was supported in part by the U.S. NSF under Grant No. PHY1620059. The work of G. T. was supported in part by the Multidisciplinary University Research Initiative (MURI) Grant No. W911NF-14-1-0003 from U.S. Army Research Office (ARO) and by DOE Grant No. de-sc0007870.

\section{APPENDIX A: THE EIGENVALUES OF THE QUADRATIC CASIMIR OPERATOR}

In this Appendix we describe the value of quadratic Casimir operator for the representations of $O(N)$ and $S U(N)$ groups in terms of Young diagrams. To extract the irreducible representation corresponding to a Young diagram from a generic tensor, we first fill in the boxes with this tensor indices, then we symmetrize over the indexes in the rows and after that antisymmetrize the indexes in the columns. In the case of the orthogonal group we additionally subtract all possible traces from the tensor.

For the representation of the group $O(N)$ that is described by the Young diagram $Y$ with row lengths $\lambda_{i}$, the quadratic Casimir operator is equal to

$$
C_{2}^{O(N), Y}=\sum_{i=1}^{\lfloor N / 2\rfloor} \lambda_{i}\left(\lambda_{i}+N-2 i\right)
$$

The dimension of this representation reads as:

$\operatorname{dim}_{\lambda}=\frac{1}{h_{\lambda}} \prod_{i=1}^{k} \frac{\left(\lambda_{i}+N-k-i-1\right) !}{(N-i) !} \prod_{j=1}^{i}\left(\lambda_{i}+\lambda_{j}+N-i-j\right)$

where $h_{\lambda}$ is the product of all hook lengths. For each box the hook length is defined as:

(hook length $)=($ number of boxes to the right $)$

$$
+(\text { number of boxes below })+1
$$

The following lemma will be useful for studying the matrix models. Let us consider two groups $O(2 n)$ and $O(2 m)$ and Young diagram $Y_{n}$ for group $O(2 n)$ such that the length of the rows is less then $m$. There is a maximal Young diagram-a rectangular $n \times m$, that we shall denote as $Y_{n \times m}$. We would like to consider a specific Young diagram $Y_{m}=\left(Y_{n \times m} / Y_{n}\right)^{T}$ for a group $O(2 m)$, where T stands for transposition. Then

$$
C_{2}^{Y_{n}}+C_{2}^{Y_{m}}=n^{2} m+n m^{2}-n m .
$$

The proof goes as follows. Let $\lambda_{i}$ be the length of rows of the diagram $Y_{n}$, we introduce $\lambda_{0}=m, \lambda_{n+1}=0$. Then

$$
C_{2}^{Y_{n}}=\sum_{i=1}^{n} \lambda_{i}\left(\lambda_{i}+2(n-i)\right)
$$

The value of Casimir operator of $C_{2}^{Y_{m}}$ can be expressed as the following. The difference $\lambda_{i}-\lambda_{i+1}$ is just equal to the number of the rows that has length $n-i$. Then

$$
\begin{aligned}
C_{2}^{Y_{m}}= & \sum_{i=0}^{n}\left[\left(\lambda_{i}-\lambda_{i+1}\right)(n-i)^{2}\right. \\
& \left.+(n-i)\left(\lambda_{i}^{2}-\lambda_{i+1}^{2}-\lambda_{i}+\lambda_{i+1}\right)\right]
\end{aligned}
$$

After that it is easy to see

$C_{2}^{Y_{m}}=m n^{2}+n m^{2}-n m-\sum_{i=0}^{n} \lambda_{i}\left(\lambda_{i}+2(n-i)\right)$

So eventually it gives us

$$
C_{2}^{Y_{m}}+C_{2}^{Y_{n}}=m n^{2}+n m^{2}-n m .
$$

We will call the representation with Young diagram $Y_{n \times m}$ to be maximal and for $O(N)$ group the dimension is $\operatorname{dim}_{\max } \sim n^{m^{2} / 2}$.

We will also need an explicit expression for the quadratic Casimir of $S U(N)$. For a Young diagram $Y$ with row lengths $\lambda_{i}$, column lengths $\mu_{j}$ and total number of boxes $b$ it is given by:

$$
C_{2}^{S U(N), Y}=\frac{1}{2} N\left[b N+\sum \lambda_{i}^{2}-\sum \mu_{j}^{2}-\frac{b^{2}}{N}\right] .
$$

\section{APPENDIX B: EXAMPLES OF ENERGY SPECTRA IN THE MATRIX MODELS}

1. The $O\left(N_{1}\right) \times O\left(N_{2}\right)$ model for small $N_{1}, N_{2}$

Let us list the allowed representations for some low values of $N_{1}$ and $N_{2}$. For $O(2)$ we label the representations by the integer charge $Q$ so that the quadratic Casimir $C_{2}^{O(2)}=Q^{2}$; for $O(3)$ by spin $j$ so that $C_{2}^{O(3)}=j(j+1)$; for $O(4) \sim S U(2) \times S U(2)$ by spins $\left(j_{1}, j_{2}\right)$ so that $C_{2}^{O(4)}=2 j_{1}\left(j_{1}+1\right)+2 j_{2}\left(j_{2}+1\right)$.

For the $O(2) \times O(2)$ model we find 2 states with $4 E / g=-1$ with charges $( \pm 1,0)$ and 2 states with $4 E / g=$ 1 with charges $(0, \pm 1)$.

For the $O(2) \times O(3)$ model we find 6 states with $4 E / g=-1$ which have $S O(3)$ spin 1 and $S O(2)$ charges $\pm 1 / 2$; and 2 states with $4 E / g=3$ which have $S O(3)$ spin 0 and $S O(2)$ charges $\pm 3 / 2$. 
For the $O(3) \times O(3)$ model we find 8 states with $4 E / g=-3$ which have spins $(1 / 2,3 / 2)$; and 8 states with $4 E / g=3$ which have spins $(3 / 2,1 / 2)$ (note the appearance of half-integral spins which correspond to spinorial representations).

For the $O(2) \times O(4)$ model we find 6 states with $4 E / g=-2$ which have $S O(2)$ charge zero and are in the $S O(4)$ representation $(1,0)+(0,1) ; 8$ states with $E=$ 0 which have $S O(2)$ charges \pm 1 and are in the $S O(4)$ representation $(1 / 2,1 / 2)$; and 2 states with $4 E / g=6$ which have $S O(2)$ charges \pm 2 and are $S O(4)$ singlets.

For the $O(3) \times O(4)$ model we find 8 states with $4 E / g=-6$ which have $S O(3)$ spin zero and are in the $S O(4)$ representation $(3 / 2,0)+(0,3 / 2) ; 36$ states with $4 E / g=-2$ which have $S O(3)$ spin 1 and are in the $S O(4)$ representation $(1 / 2,1)+(1,1 / 2)$; and 20 states with $4 E / g=6$ which have $S O(3)$ spin 2 and are in the $S O(4)$ representation $(1 / 2,0)+(0,1 / 2)$.

For the $O(4) \times O(4)$ model we find 10 ground states with $4 E / g=-12$ which are $S O(4)_{1}$ singlets and are in the $\mathrm{SO}(4)_{2}$ representation $(2,0)+(0,2) ; 64$ states with $4 E / g=$ -6 which are in $S O(4)_{1}$ representation $(1 / 2,1 / 2)$ and in the $\mathrm{SO}(4)_{2}$ representation $(1 / 2,3 / 2)+(3 / 2,1 / 2)$; etc.

For the $O(6) \times O(6)$ model we find 84 ground states with $4 E / g=-45$ which are $S O(6)_{1}$ singlets and are in the $\mathrm{SO}(6)_{2}$ representation whose Young diagram is a $3 \times 3$ square. The first excited state has $4 E / g=-35$; it transforms as a vector of $S O(6)_{1}$ and in the representation of $\mathrm{SO}(6)_{2}$ whose Young diagram has 3 boxes in the first row, 3 in the second row, and 2 in the third row.

Due to the relation (A4) we can state the general correspondence between the representations of $O\left(N_{1}\right) \times$ $O\left(N_{2}\right)$ if $N_{1}$ and $N_{2}$ are even. If the state is described by representation $Y_{1}$ for the group $O\left(N_{1}\right)$, then it has the representation $\left(Y_{N_{1} / 2 \times N_{2} / 2} / Y_{1}\right)^{T}$ for the second group $\mathrm{O}\left(\mathrm{N}_{2}\right)$.

\section{The $O(2) \times O(3) \times U(1)$ model}

As was described in the main text, first we have to find $S U(2) \times S U(3)$ representations and then decompose into $S O(2) \times S O(3)$ irreducible representations. After that we can directly apply the exact formula (6.23) for the energy.

Let us list the explicit form of quadratic Casimirs. For $S O(2)$ the quadratic Casimir is simply $Q^{2}$, where $Q$ is the charge. For $S U(2)$ and $S O(3)$ it equals $j(j+1)$ where $j$ is spin [an integer for $S O(3)$ and half-integer for $S U(2)$ ]. For $S U(3)$ the quadratic Casimir in our normalization reads as:

$$
C_{2}^{S U(3)}(\lambda)=\frac{1}{2}\left(l_{1}^{2}+l_{2}^{2}-\frac{1}{3}\left(l_{1}+l_{2}\right)^{2}+2 l_{1}\right),
$$

where $l_{1}>l_{2}>\ldots$ are the row lengths of the Young diagram $\lambda$ defining the representation $\lambda$. For example, $C_{2}^{S U(3)}(\square)=\frac{4}{3}, \quad C_{2}^{S U(3)}(\square)=\frac{10}{3}, \quad$ and $\quad C_{2}^{S U(3)}(\square)=3$ (the last one is the adjoint representation).
TABLE VII. Energy spectrum of the $O(2) \times O(3) \times O(2)$ model. Due to the charge conjugation symmetry for the last $O(2)$ charge, the energies and representations are invariant under transformation level $\rightarrow 6-$ level.

\begin{tabular}{lccc}
\hline \hline Level & $S U(2) \times S U(3)$ irrep & $S O(2) \times S O(3)$ irrep & $\frac{4}{g}$ Energy \\
\hline 0 & $\varnothing \times \varnothing$ & $\varnothing \times \varnothing$ & 3 \\
1 & $\square \times \square$ & $\square \times \square$ & 1 \\
2 & $\square \times \square$ & $\square \times \square$ & -1 \\
2 & $\varnothing \times \square$ & 7 \\
2 & $\varnothing \times \square \square$ & $\varnothing \times \square$ & -1 \\
2 & $\square \times \square \square$ & $\square \times \square$ & -13 \\
3 & $\square \times \varnothing$ & 1 \\
3 & $\square \times \varnothing$ & $\square \square \times \varnothing$ & -7 \\
3 & $\square \times \square \square$ & $\square \times \varnothing$ & -3 \\
3 & $\varnothing \times \square$ & 13 \\
4 & $\square \times \square$ & $\varnothing \times \square$ & -13 \\
4 & $\square \times \square$ & $\square \times \square$ & 7 \\
4 & $\square \times \varnothing$ & $\varnothing \times \varnothing$ & -1 \\
4 & $\square \times \square$ & 1 \\
5 & $\square$ & & 3 \\
6 & $\square$ & $\square \times \square$ & \\
\hline \hline
\end{tabular}

The spectrum can be found in Table VII; it coincides with the one in Table VI.

\section{Explicit form of some singlet states}

The construction of singlet states for the $O\left(N_{1}\right) \times$ $O\left(N_{2}\right) \times O\left(N_{3}\right)$ tensor quantum mechanics is in general a difficult problem, but it simplifies when one of the groups is $O(2)$. The singlet states, which exist only when $N_{1}$ and $\mathrm{N}_{2}$ are even, may sometimes be written down by inspection in the oscillator basis. In this basis, in addition to the manifest $S O\left(N_{1}\right) \times S O\left(N_{2}\right)$ symmetry, there is manifest discrete $Z_{2} \times Z_{2}$ parity symmetry contained inside $O\left(N_{1}\right) \times O\left(N_{2}\right)$.

For example, for the $O(2)^{3}$ model there are only two singlet states

$\epsilon_{a_{1} a_{2}} \delta_{b_{1} b_{2}} \bar{\psi}_{a_{1} b_{1}} \bar{\psi}_{a_{2} b_{2}}|0\rangle, \quad \epsilon_{b_{1} b_{2}} \delta_{a_{1} a_{2}} \bar{\psi}_{a_{1} b_{1}} \bar{\psi}_{a_{2} b_{2}}|0\rangle$,

since due to the Fermi statistics the other two invariant contractions vanish. Under the $Z_{2} \times Z_{2}$ symmetry these states are $(-,+)$ and $(+,-)$, respectively. In agreement with section VIC, one of these states is invariant under $S U(2) \times S O(2) \times S O(2)$, while the other under $S O(2) \times S U(2) \times S O(2)$.

Generalizing to any $O\left(N_{1}\right) \times O(2)^{2}$ model with even $N_{1}$, we again find only two singlet states. They may be written as

$\epsilon_{a_{1}, \ldots a_{N_{1}}} \delta_{b_{1} b_{2}} \ldots \delta_{b_{N_{1}-1} b_{N_{1}}} \bar{\psi}_{a_{1} b_{1}} \ldots \bar{\psi}_{a_{N_{1}} b_{N_{1}}}|0\rangle$,

$\left(\epsilon_{b_{1} b_{2}} \delta_{a_{1} a_{2}} \bar{\psi}_{a_{1} b_{1}} \bar{\psi}_{a_{2} b_{2}}\right)^{N_{1} / 2}|0\rangle$.

One of these states is invariant under $S U\left(N_{1}\right) \times S O(2) \times$ $S O(2)$, while the other under $S O\left(N_{1}\right) \times S U(2) \times S O(2)$. 
For the $O(4)^{2} \times O(2)$ model there are 4 singlet states

$$
\begin{aligned}
& \epsilon_{a_{1} a_{2} a_{3} a_{4}} \epsilon_{a_{5} a_{6} a_{7} a_{8}} \delta_{b_{1} b_{5}} \ldots \delta_{b_{4} b_{8}} \bar{\psi}_{a_{1} b_{1}} \ldots \bar{\psi}_{a_{8} b_{8}}|0\rangle, \\
& \epsilon_{b_{1} b_{2} b_{3} b_{4}} \epsilon_{b_{5} b_{6} b_{7} b_{8}} \delta_{a_{1} a_{5}} \ldots \delta_{a_{4} a_{8}} \bar{\psi}_{a_{1} b_{1}} \ldots \bar{\psi}_{a_{8} b_{8}}|0\rangle, \\
& \left(\epsilon_{a_{1} a_{2} a_{3} a_{4}} \delta_{b_{1} b_{2}} \delta_{b_{3} b_{4}} \bar{\psi}_{a_{1} b_{1}} \ldots \bar{\psi}_{a_{4} b_{4}}\right) \\
& \quad \times\left(\delta_{a_{5} a_{6}} \delta_{a_{7} a_{8}} \delta_{b_{5} b_{7}} \delta_{b_{6} b_{8}} \bar{\psi}_{a_{5} b_{5}} \ldots \bar{\psi}_{a_{8} b_{8}}\right)|0\rangle, \\
& \left(\epsilon_{b_{1} b_{2} b_{3} b_{4}} \delta_{a_{1} a_{2}} \delta_{a_{3} a_{4}} \bar{\psi}_{a_{1} b_{1}} \ldots \bar{\psi}_{a_{4} b_{4}}\right) \\
& \quad \times\left(\delta_{b_{5} b_{6}} \delta_{b_{7} b_{8}} \delta_{a_{5} a_{7}} \delta_{a_{6} a_{8}} \bar{\psi}_{a_{5} b_{5}} \ldots \bar{\psi}_{a_{8} b_{8}}\right)|0\rangle .
\end{aligned}
$$

The first pair of states have energies $E= \pm 16 g$, saturating the energy bound (3.10). One of these states is invariant under $S U(4) \times O(4) \times O(2)$, while the other under $O(4) \times S U(4) \times O(2)$. The second pair of states have energies $E= \pm 4 g$.

Defining the antisymmetric matrix $M_{b_{1} b_{2}}=\bar{\psi}_{a b_{1}} \bar{\psi}_{a b_{2}}$, we can write the first two states as

$$
\left(\operatorname{tr} M^{4} \pm \frac{1}{2}\left(\operatorname{tr} M^{2}\right)^{2}\right)|0\rangle
$$

By analogy with (B5), for $N$ a multiple of 4 we may build a set of states by acting on $|0\rangle$ with traces of powers of $M$. For example, for $N=8$ we can act with $\operatorname{tr} M^{16}$, $\operatorname{tr} M^{2} \operatorname{tr} M^{14}$, etc. The number of such terms is $P(8)$, i.e. the number of partitions of 8 into positive integers, and $P(8)=22$. For $O(12)^{2} \times O(2)$ the number of such terms is $P(18)=385$. However, these terms are not linearly independent, so this should be regarded as an upper bound on the number of invariant states.

More generally, for $O(N)^{2} \times O(2)$ with $N$ a multiple of 4, this upper bound is $P\left(N^{2} / 8\right)$, which grows exponentially with $N$ :

$$
P\left(N^{2} / 8\right) \rightarrow \frac{2}{N^{2} \sqrt{3}} \exp \left(\frac{\pi N}{2 \sqrt{3}}\right)
$$

[1] E. Witten, An SYK-like model without disorder, arXiv: 1610.09758.

[2] I. R. Klebanov and G. Tarnopolsky, Uncolored random tensors, melon diagrams, and the Sachdev-Ye-Kitaev models, Phys. Rev. D 95, 046004 (2017).

[3] R. Gurau, Colored group field theory, Commun. Math. Phys. 304, 69 (2011).

[4] R. Gurau and V. Rivasseau, The 1/N expansion of colored tensor models in arbitrary dimension, Europhys. Lett. 95, 50004 (2011).

[5] R. Gurau, The complete 1/N expansion of colored tensor models in arbitrary dimension, Ann. Henri Poincare 13, 399 (2012).

[6] V. Bonzom, R. Gurau, A. Riello, and V. Rivasseau, Critical behavior of colored tensor models in the large N limit, Nucl. Phys. B853, 174 (2011).

[7] A. Tanasa, Multi-orientable group field theory, J. Phys. A 45, 165401 (2012).

[8] V. Bonzom, R. Gurau, and V. Rivasseau, Random tensor models in the large $\mathrm{N}$ limit: Uncoloring the colored tensor models, Phys. Rev. D 85, 084037 (2012).

[9] S. Dartois, V. Rivasseau, and A. Tanasa, The $1 / N$ expansion of multi-orientable random tensor models, Ann. Henri Poincare 15, 965 (2014).

[10] S. Carrozza and A. Tanasa, $O(N)$ random tensor models, Lett. Math. Phys. 106, 1531 (2016).

[11] R. Gurau, The complete $1 / N$ expansion of a SYK-like tensor model, Nucl. Phys. B916, 386 (2017).

[12] R. Gurau and J. P. Ryan, Colored tensor models-a review, SIGMA 8, 020 (2012).

[13] A. Tanasa, The multi-orientable random tensor model, a review, SIGMA 12, 056 (2016).
[14] R. Gurau, Invitation to random tensors, SIGMA 12, 094 (2016).

[15] A. Almheiri and J. Polchinski, Models of $\mathrm{AdS}_{2}$ backreaction and holography, J. High Energy Phys. 11 (2015) 014.

[16] J. Maldacena, D. Stanford, and Z. Yang, Conformal symmetry and its breaking in two dimensional nearly antide-Sitter space, Prog. Theor. Exp. Phys. (2016) $12 \mathrm{C} 104$.

[17] J. Engelsoy, T. G. Mertens, and H. Verlinde, An investigation of $\mathrm{AdS}_{2}$ backreaction and holography, J. High Energy Phys. 07 (2016) 139.

[18] K. Jensen, Chaos in AdS $_{2}$ Holography, Phys. Rev. Lett. 117, 111601 (2016).

[19] J. M. Maldacena, The large N limit of superconformal field theories and supergravity, Int. J. Theor. Phys. 38, 1113 (1999); Adv. Theor. Math. Phys. 2, 231 (1998).

[20] S. S. Gubser, I. R. Klebanov, and A. M. Polyakov, Gauge theory correlators from noncritical string theory, Phys. Lett. B 428, 105 (1998).

[21] E. Witten, Anti-de Sitter space and holography, Adv. Theor. Math. Phys. 2, 253 (1998).

[22] S. Sachdev and J. Ye, Gapless Spin Fluid Ground State in a Random, Quantum Heisenberg Magnet, Phys. Rev. Lett. 70, 3339 (1993).

[23] O. Parcollet and A. Georges, Non-Fermi-liquid regime of a doped Mott insulator, Phys. Rev. B 59, 5341 (1999).

[24] A. Georges, O. Parcollet, and S. Sachdev, Mean Field Theory of a Quantum Heisenberg Spin Glass, Phys. Rev. Lett. 85, 840 (2000).

[25] A. Kitaev, A simple model of quantum holography, http://online.kitp.ucsb.edu/online/entangled15/kitaev/, http://online.kitp.ucsb.edu/online/entangled15/kitaev2/. Talks at KITP, April 7, 2015 and May 27, 2015. 
[26] J. Polchinski and V. Rosenhaus, The spectrum in the Sachdev-Ye-Kitaev model, J. High Energy Phys. 04 (2016) 001.

[27] J. Maldacena and D. Stanford, Comments on the SachdevYe-Kitaev model, Phys. Rev. D 94, 106002 (2016).

[28] A. Jevicki, K. Suzuki, and J. Yoon, Bi-local holography in the SYK model, J. High Energy Phys. 07 (2016) 007.

[29] D. J. Gross and V. Rosenhaus, A generalization of SachdevYe-Kitaev, J. High Energy Phys. 02 (2017) 093.

[30] V. Bonzom, L. Lionni, and A. Tanasa, Diagrammatics of a colored SYK model and of an SYK-like tensor model, leading and next-to-leading orders, J. Math. Phys. (N.Y.) 58, 052301 (2017).

[31] I. R. Klebanov and G. Tarnopolsky, On large $N$ limit of symmetric traceless tensor models, J. High Energy Phys. 10 (2017) 037.

[32] F. Ferrari, V. Rivasseau, and G. Valette, A new large N expansion for general matrix-tensor models, arXiv: 1709.07366 .

[33] D. Benedetti and R. Gurau, 2PI effective action for the SYK model and tensor field theories, arXiv:1802.05500.

[34] K. Bulycheva, I. R. Klebanov, A. Milekhin, and G. Tarnopolsky, Spectra of operators in large $N$ tensor models, Phys. Rev. D 97, 026016 (2018).

[35] S. Choudhury, A. Dey, I. Halder, L. Janagal, S. Minwalla, and R. Poojary, Notes on Melonic $S O(N)^{q-1}$ Tensor Models, arXiv:1707.09352.

[36] M. Beccaria and A. A. Tseytlin, Partition function of free conformal fields in 3-plet representation, J. High Energy Phys. 05 (2017) 053.

[37] S. Sachdev, Bekenstein-Hawking Entropy and Strange Metals, Phys. Rev. X 5, 041025 (2015).

[38] A. M. Garca-Garca and J. J. M. Verbaarschot, Spectral and thermodynamic properties of the Sachdev-Ye-Kitaev model, Phys. Rev. D 94, 126010 (2016).

[39] J. S. Cotler, G. Gur-Ari, M. Hanada, J. Polchinski, P. Saad, S. H. Shenker, D. Stanford, A. Streicher, and M. Tezuka, Black holes and random matrices, J. High Energy Phys. 05 (2017) 118.
[40] C. Krishnan, S. Sanyal, and P. N. Bala Subramanian, Quantum chaos and holographic tensor models, J. High Energy Phys. 03 (2017) 056.

[41] I. Klebanov, D. Roberts, D. Stanford, and G. Tarnopolsky (unpublished).

[42] C. Krishnan, K. V. P. Kumar, and S. Sanyal, Random matrices and holographic tensor models, J. High Energy Phys. 06 (2017) 036.

[43] S. Chaudhuri, V. I. Giraldo-Rivera, A. Joseph, R. Loganayagam, and J. Yoon, Abelian tensor models on the lattice, Phys. Rev. D 97, 086007 (2018).

[44] C. Krishnan and K. V. P. Kumar, Towards a finite- $N$ hologram, J. High Energy Phys. 10 (2017) 099.

[45] C. Krishnan, K. V. Pavan Kumar, and D. Rosa, Contrasting SYK-like models, J. High Energy Phys. 01 (2018) 064.

[46] C. Krishnan and K. V. Pavan Kumar, Exact solution of a strongly coupled gauge theory in $0+1$ dimensions, arXiv: 1802.02502.

[47] F. Ferrari, The large D limit of planar diagrams, arXiv: 1701.01171.

[48] T. Azeyanagi, F. Ferrari, P. Gregori, L. Leduc, and G. Valette, More on the new large $D$ limit of matrix models, arXiv:1710.07263.

[49] D. Anninos and G. A. Silva, Solvable quantum Grassmann matrices, J. Stat. Mech. 04 (2017) 043102.

[50] A. Kitaev, Periodic table for topological insulators and superconductors, AIP Conf. Proc. 1134, 22 (2009).

[51] J. Yoon, SYK models and SYK-like tensor models with global symmetry, J. High Energy Phys. 10 (2017) 183.

[52] O. Aharony, J. Marsano, S. Minwalla, K. Papadodimas, and M. Van Raamsdonk, The Hagedorn-deconfinement phase transition in weakly coupled large $\mathrm{N}$ gauge theories, Adv. Theor. Math. Phys. 8, 603 (2004).

[53] S. Elitzur, Y. Frishman, E. Rabinovici, and A. Schwimmer, Origins of global anomalies in quantum mechanics, Nucl. Phys. B273, 93 (1986).

[54] M. Tierz, Polynomial solution of quantum Grassmann matrices, J. Stat. Mech. 05 (2017) 053203.

[55] D. Anninos, F. Denef, and R. Monten, Grassmann matrix quantum mechanics, J. High Energy Phys. 04 (2016) 138. 